\title{
Pseudospectral functions of various dimensions for symmetric systems with the maximal deficiency index
}

\author{
VADIM MogILEvSKII
}

(Presented by M.M. Malamud)

\begin{abstract}
We consider first-order symmetric system $J y^{\prime}-A(t) y=$ $\lambda \Delta(t) y$ with $n \times n$-matrix coefficients defined on an interval $[a, b)$ with the regular endpoint $a$. It is assumed that the deficiency indices $N_{ \pm}$of the system satisfies $N_{-} \leq N_{+}=n$. The main result is a parametrization of all pseudospectral functions $\sigma(\cdot)$ of any possible dimension $n_{\sigma} \leq$ $n$ by means of a Nevanlinna parameter $\tau=\left\{C_{0}(\lambda), C_{1}(\lambda)\right\}$. Such a parametrization is given by the linear-fractional transform

$$
m_{\tau}(\lambda)=\left(C_{0}(\lambda) w_{11}(\lambda)+C_{1}(\lambda) w_{21}(\lambda)\right)^{-1}\left(C_{0}(\lambda) w_{12}(\lambda)+C_{1}(\lambda) w_{22}(\lambda)\right)
$$

and the Stieltjes inversion formula for $m_{\tau}(\lambda)$. We also show that the matrix $W(\lambda)=\left(w_{i j}(\lambda)\right)_{i, j=1}^{2}$ has the properties similar to those of the resolvent matrix in the extension theory of symmetric operators. The obtained results develop the results by A. Sakhnovich; Arov and Dym; Langer and Textorius.
\end{abstract}

2010 MSC. 34L10,47A06,47E05.

Key words and phrases. Symmetric differential system, pseudospectral function, spectral function, Fourier transform, monodromy matrix.

\section{Introduction}

Let $\mathbb{H}$ be a finite dimensional Hilbert space, let $n=\operatorname{dim} \mathbb{H}$ and let $[\mathbb{H}]$ be the set of linear operators in $\mathbb{H}$. We consider symmetric differential system $[6,17]$

$$
J y^{\prime}-A(t) y=\lambda \Delta(t) y, \quad t \in \mathcal{I}, \quad \lambda \in \mathbb{C},
$$

where $J \in[\mathbb{H}], J^{*}=J^{-1}=-J$ and $A(t)=A^{*}(t)$ and $\Delta(t) \geq 0$ are $[\mathbb{H}]$-valued locally-integrable functions defined on an interval $\mathcal{I}=$ $[a, b),-\infty<a<b \leq \infty$.

Received 07.06.2017 
Let for definiteness $\operatorname{dim} \operatorname{ker}(J-i I) \geq \operatorname{dim} \operatorname{ker}(J+i I)$. Then without loss of generality one may assume that $\mathbb{H}=H \oplus \widehat{H} \oplus H$ with finitedimensional Hilbert spaces $H$ and $\widehat{H}$ and that

$$
J=\left(\begin{array}{ccc}
0 & 0 & -I_{H} \\
0 & i I_{\widehat{H}} & 0 \\
I_{H} & 0 & 0
\end{array}\right): H \oplus \widehat{H} \oplus H \rightarrow H \oplus \widehat{H} \oplus H .
$$

System (1.1) is called a Hamiltonian system if $\widehat{H}=\{0\}$ and hence $\mathbb{H}=$ $H \oplus H$,

$$
J=\left(\begin{array}{cc}
0 & -I_{H} \\
I_{H} & 0
\end{array}\right): H \oplus H \rightarrow H \oplus H .
$$

Recall that system (1.1) is called regular if $b<\infty$ and $\int_{\mathcal{I}}\|A(t)\| d t<$ $\infty, \quad \int_{\mathcal{I}}\|\Delta(t)\| d t<\infty$; otherwise it is called singular. Pseudospectral functions of regular Hamiltonian systems were studied in $[3,5,36$, 37]. Namely, denote by $\mathfrak{H}=L_{\Delta}^{2}(\mathcal{I})$ the Hilbert space of functions $f: \mathcal{I} \rightarrow \mathbb{H}$ satisfying $\int_{\mathcal{I}}(\Delta(t) f(t), f(t)) d t<\infty$. Let $Y(\cdot, \lambda)$ be the $[\mathbb{H}]-$ valued operator solution of (1.1) with $Y(a, \lambda)=I_{\mathbb{H}}$ and let $Y(t, \lambda)=$ $(\varphi(t, \lambda), \psi(t, \lambda))(\in H \oplus H, \mathbb{H})$ be the block representation of $Y(t, \lambda)$. Then according to [36] an $[H]$-valued operator (matrix) distribution function $\sigma(\cdot): \mathbb{R} \rightarrow[H]$ is called a pseudospectral function of the regular Hamiltonian system (1.1) if the (generalized) Fourier transform $V_{\sigma}: \mathfrak{H} \rightarrow$ $L^{2}(\sigma ; H)$ defined by

$$
\left(V_{\sigma} f\right)(s)=\widehat{f}(s):=\int_{\mathcal{I}} \varphi^{*}(t, s) \Delta(t) f(t) d t, \quad f(\cdot) \in \mathfrak{H}
$$

is a partial isometry with the minimally possible kernel $\operatorname{ker} V_{\sigma}=L_{0}:=$ $\{f \in \mathfrak{H}: \widehat{f}(s)=0, s \in \mathbb{R}\}$. Moreover, $\sigma(\cdot)$ is a spectral function if $V_{\sigma}$ is an isometry. Clearly the dimension $n_{\sigma}$ of the matrix $\sigma(s)$ is $n_{\sigma}=\operatorname{dim} H$.

A description of all pseudospectral functions is specified in the following theorem obtained by A. L. Sakhnovich in [36] (see also [37]).

Theorem 1.1. Let system (1.1) be regular and Hamiltonian with $A(t) \equiv$ 0 , let

$$
W(\lambda)=\left(\begin{array}{ll}
w_{1}(\lambda) & w_{2}(\lambda) \\
w_{3}(\lambda) & w_{4}(\lambda)
\end{array}\right): H \oplus H \rightarrow H \oplus H, \quad \lambda \in \mathbb{C}
$$

be the block representation of the monodromy matrix $W(\lambda):=Y(b, \lambda)$ and let $\bigcap_{\lambda \in \mathbb{C}} \operatorname{ker} w_{1}(\lambda)=\{0\}$. Then for each Nevanlinna pair $\tau=\left\{C_{0}(\lambda), C_{1}(\lambda)\right\}$, 
$C_{j}(\lambda) \in[H], \lambda \in \mathbb{C}_{+}, j \in\{0,1\}$, satisfying a certain admissibility condition the equalities

$$
\begin{gathered}
m(\lambda)=\left(C_{0}(\lambda) w_{1}(\lambda)+C_{1}(\lambda) w_{3}(\lambda)\right)^{-1} \\
\times\left(C_{0}(\lambda) w_{2}(\lambda)+C_{1}(\lambda) w_{4}(\lambda)\right), \quad \lambda \in \mathbb{C}_{+} \\
\sigma(s)=\lim _{\delta \rightarrow+0} \lim _{\varepsilon \rightarrow+0} \frac{1}{\pi} \int_{-\delta}^{s-\delta} \operatorname{Im} m(u+i \varepsilon) d u
\end{gathered}
$$

defines a pseudospectral function $\sigma(\cdot)$ and, conversely, each pseudospectral function $\sigma(\cdot)$ is defined by (1.5), (1.6) with some admissible Nevanlinna pair $\tau=\left\{C_{0}(\lambda), C_{1}(\lambda)\right\}$. Moreover, in the case $L_{0}=\{0\}$ (and only in this case) the set of spectral functions is not empty and the above statement holds for spectral functions.

It was also shown by D.Z. Arov and H. Dym in $[3,5]$ that under certain additional conditions on $W(\lambda)$ statements of Theorem 1.1 hold with arbitrary (not necessarily admissible) Nevanlinna pairs $\tau$ and the correspondence between $\sigma(\cdot)$ and $\tau$ is one to one.

The above results on pseudospectral functions were developed in our papers [32-35]. Namely, in [32,35] definitions of pseudospectral and spectral functions $\sigma(\cdot)$ were extended to general (regular or singular) possibly non-Hamiltonian system (1.1), (1.2) (see Definition 3.2 below). It is proved in [35] that under the natural additional conditions the dimension $n_{\sigma}$ of $\sigma(\cdot)$ satisfies $\operatorname{dim} H+\operatorname{dim} \widehat{H} \leq n_{\sigma} \leq n$.

Denote by $\mathcal{N}_{\lambda}$ the linear space of solutions of (1.1) belonging to $\mathfrak{H}$ and let $N_{ \pm}=\operatorname{dim} \mathcal{N}_{\lambda}, \lambda \in \mathbb{C}_{ \pm}$, be the deficiency indices of the system. In [3235] statements of Theorem 1.1 were extended to pseudospectral functions $\sigma(\cdot)$ of any possible dimension $n_{\sigma}$ for arbitrary (possibly singular and non-Hamiltonian) symmetric system (1.1), (1.2) with arbitrary (possibly unequal) deficiency indices $N_{ \pm}$. In particular, according to [35] (see also Theorem 3.12 below) the parametrization of all pseudospectral functions $\sigma(\cdot)$ of a fixed dimension $n_{\sigma}$ is given by the Redheffer transform

$$
m(\lambda)=m_{0}(\lambda)+S_{1}(\lambda)\left(C_{0}(\lambda)-C_{1}(\lambda) \dot{\mathcal{M}}(\lambda)\right)^{-1} C_{1}(\lambda) S_{2}(\lambda), \quad \lambda \in \mathbb{C}_{+}
$$

of the Nevanlinna parameter $\tau=\left\{C_{0}(\lambda), C_{1}(\lambda)\right\}$ and by formula (1.6) for the (Nevanlinna) operator function $m(\cdot)$, which is the Titchmarsh-Weyl function of the system. The operator coefficients $m_{0}, S_{1}, S_{2}$ and $\dot{\mathcal{M}}$ in (1.7) are defined in terms of the boundary values of respective operator solutions of (1.1) at the endpoints $a$ and $b$.

In the present paper we study pseudospectral and spectral functions of symmetric system (1.1), (1.2) with the maximal deficiency index $N_{+}=n$ 
and an arbitrary deficiency index $N_{-}$. The partial case here is a quasiregular system, i.e., the system with $N_{+}=N_{-}=n$ (clearly, each regular system is quasiregular). For a system with $N_{+}=n$ we define the monodromy matrix $B(\lambda)(\in[\mathbb{H}]), \lambda \in \mathbb{C}_{+}$, as a singular boundary value of $Y(\cdot, \lambda)$ at the endpoint $b$. The main result of the paper is a parametrization of all pseudospectral and spectral functions $\sigma(\cdot)$ of a given dimension $n_{\sigma}$ for general system (1.1), (1.2) with $N_{-} \leq N_{+}=n$. Unlike (1.7) such a parametrization is given by the linear-fractional transform (1.5) with the operator-valued coefficients $w_{j}(\lambda)$ defined in terms of $B(\lambda)$ and by formula (1.6). In the simplest case of the minimal $n_{\sigma}=\operatorname{dim} H+\operatorname{dim} \widehat{H}$ this result can be formulated as the following theorem.

Theorem 1.2. Let for simplicity system (1.1), (1.2) be quasiregular. Assume that there exists only a trivial solution $y=0$ of this system such that $\Delta(t) y(t)=0$ (a.e. on I) and $y(a) \in H \oplus\{0\} \oplus\{0\}$. Let $B(\lambda)=$ $\left\{B_{i j}(\lambda)\right\}_{i, j=1}^{3}$ be the block representation of the monodromy matrix and let $W(\lambda)=\left(\begin{array}{ll}w_{1}(\lambda) & w_{2}(\lambda) \\ w_{3}(\lambda) & w_{4}(\lambda)\end{array}\right)$, where $w_{j}(\lambda) \in[H \oplus \widehat{H}], \lambda \in \mathbb{C}_{+}$, are defined by

$$
\begin{gathered}
w_{1}(\lambda)=\left(\begin{array}{cc}
B_{11} & B_{12} \\
-i B_{21} & -i\left(B_{22}-I_{\widehat{H}}\right)
\end{array}\right), w_{2}(\lambda)=\left(\begin{array}{cc}
B_{13} & \frac{i}{2} B_{12} \\
-i B_{23} & \frac{1}{2}\left(B_{22}+I_{\widehat{H}}\right)
\end{array}\right) \\
w_{3}(\lambda)=\left(\begin{array}{cc}
B_{31} & B_{32} \\
-\frac{1}{2} B_{21} & -\frac{1}{2}\left(B_{22}+I_{\widehat{H}}\right)
\end{array}\right), \\
w_{4}(\lambda)=\left(\begin{array}{cc}
B_{33} & \frac{i}{2} B_{32} \\
-\frac{1}{2} B_{23} & -\frac{i}{4}\left(B_{22}-I_{\widehat{H}}\right)
\end{array}\right)
\end{gathered}
$$

with $B_{i j}=B_{i j}(\lambda)$ (clearly for the Hamiltonian system $W(\lambda)=B(\lambda)$ ). Then:

(1) The equality

$$
\begin{gathered}
m(\lambda)=\left(C_{0}(\lambda) w_{1}(\lambda)+C_{1}(\lambda) w_{3}(\lambda)\right)^{-1} \\
\times\left(C_{0}(\lambda) w_{2}(\lambda)+C_{1}(\lambda) w_{4}(\lambda)\right), \quad \lambda \in \mathbb{C}_{+}
\end{gathered}
$$

together with (1.6) establishes a bijective correspondence between all $\mathrm{Ne}$ vanlinna pairs $\tau=\left\{C_{0}(\lambda), C_{1}(\lambda)\right\}, C_{j}(\lambda) \in[H \oplus \widehat{H}], j \in\{0,1\}$, satisfying the admissibility conditions

$$
\begin{aligned}
& \lim _{y \rightarrow+\infty} \frac{1}{i y} w_{1}(i y)\left(C_{0}(i y) w_{1}(i y)+C_{1}(i y) w_{3}(i y)\right)^{-1} C_{1}(i y)=0 \\
& \lim _{y \rightarrow+\infty} \frac{1}{i y} w_{3}(i y)\left(C_{0}(i y) w_{1}(i y)+C_{1}(i y) w_{3}(i y)\right)^{-1} C_{0}(i y)=0,
\end{aligned}
$$


and all pseudospectral functions $\sigma(s)(\in[H \oplus \widehat{H}])$ of the dimension $n_{\sigma}=$ $\operatorname{dim} H+\operatorname{dim} \widehat{H}$.

(2) Conditions (1.11) and (1.12) can be omitted if and only if

$$
\lim _{y \rightarrow+\infty} y(\|h\|-\|\chi(i y) h\|)=+\infty, \quad 0 \neq h \in H \oplus \widehat{H} .
$$

Here $\chi(\cdot)$ is a contractive operator function defined by

$$
\chi(\lambda)=\left(w_{3}(\lambda)+i w_{1}(\lambda)\right)\left(w_{3}(\lambda)-i w_{1}(\lambda)\right)^{-1}, \quad \lambda \in \mathbb{C}_{+} .
$$

(3) The set of spectral functions $\sigma(s)(\in[H \oplus \widehat{H}])$ either is empty or coincides with the set of pseudospectral functions. In the latter case statements (1) and (2) hold for spectral functions instead of pseudospectral ones.

Actually statements of Theorem 1.2 with the slightly modified conditions (1.11) and (1.12) are valid for systems (1.1) with $N_{-} \leq N_{+}=n$ (see Theorem 4.17 below). In the particular case of the quasiregular Hamiltonian system Theorem 1.2 was proved in [34].

In the case of the regular system one has $B(\lambda)=Y(b, \lambda)$. Therefore for regular Hamiltonian systems (1.1) with $A(t) \equiv 0$ Theorem 1.2 yields Theorem 1.1.

Note that admissibility conditions (1.11) and (1.12) are essentially simpler than the similar condition in $[36,37]$; actually they are consequences of $M$-admissibility conditions for symmetric operators [9,10,31]. Observe also that statement (2) of Theorem 1.2 is stronger than similar result from $[3,5]$ mentioned just after Theorem 1.1. More detailed comparison of our results for Hamiltonian systems with those from $[3,5,36,37]$ can be found in [34].

As is known (see e.g. [5]) for a regular Hamiltonian system the monodromy matrix $W(\lambda)(=B(\lambda))$ is an entire $i J$-inner operator-function. This fact enables the authors of $[5,36]$ to apply the method based on the theory of reproducing kernel Hilbert spaces associated with entire $i J$-inner matrix functions. At the same time there exist singular systems with $N_{-} \leq N_{+}=n$ for which $W(\lambda)$ is not $i J$-inner function and hence the method of $[5,36]$ is not applicable to such systems. Therefore our approach based on the extension theory of symmetric linear relations seems to be more convenient for studying of pseudospectral functions of singular symmetric systems.

Existence of scalar pseudospectral functions for the Hamiltonian system (1.1) in the case $\operatorname{dim} H=1$ was proved by I. S. Kats (see [20] and references therein). Existence of pseudospectral functions $\sigma(\cdot)$ of the maximal dimension $n_{\sigma}=n$ was proved in $[13,14,25,26]$. In $[25,26]$ 
a parametrization of all pseudospectral functions $\sigma(\cdot)$ with $n_{\sigma}=n$ for regular system (1.1) is given in the form close to (1.2), (1.6) (similar parametrization follows also from [3, Theorem 4.3]). Close result for system (1.1), (1.2) with $N_{-} \leq N_{+}=n$ is obtained in our paper [33].

In the forthcoming paper we are going to apply the obtained results to studying of spectral functions for the vector-valued Fourier transform.

\section{Preliminaries}

\subsection{Notations}

The following notations will be used throughout the paper: $\mathfrak{H}, \mathcal{H}$ denote separable Hilbert spaces; $\left[\mathcal{H}_{1}, \mathcal{H}_{2}\right]$ is the set of all bounded linear operators defined on $\mathcal{H}_{1}$ with values in $\mathcal{H}_{2} ;[\mathcal{H}]:=[\mathcal{H}, \mathcal{H}] ; \mathbb{C}_{+}\left(\mathbb{C}_{-}\right)$is the upper (lower) half-plane of the complex plane. If $\mathcal{H}$ is a subspace in $\widetilde{\mathcal{H}}$, then $P_{\mathcal{H}}(\in[\widetilde{\mathcal{H}}])$ denote the orthoprojection in $\widetilde{\mathcal{H}}$ onto $\mathcal{H}$ and $P_{\widetilde{\mathcal{H}}, \mathcal{H}}(\in$ $[\widetilde{\mathcal{H}}, \mathcal{H}])$ is the same orthoprojection considered as an operator from $\widetilde{\mathcal{H}}$ to $\mathcal{H}$.

In the following $\mathbf{C}\left[\mathcal{H}_{0}, \mathcal{H}_{1}\right]$ is the set of all holomorphic operatorfunctions $K(\cdot): \mathbb{C}_{+} \rightarrow\left[\mathcal{H}_{0}, \mathcal{H}_{1}\right]$ such that $\|K(\lambda)\| \leq 1$ for all $\lambda \in \mathbb{C}_{+}$, $\mathbf{C}[\mathcal{H}]:=\mathbf{C}[\mathcal{H}, \mathcal{H}]$ and $\mathbf{C}$ is the set of all $\mathbb{C}$-valued functions $K(\lambda)$ holomorphic on $\mathbb{C}_{+}$and satisfying $|K(\lambda)| \leq 1, \lambda \in \mathbb{C}_{+}$.

Recall that a linear relation $T: \mathcal{H}_{0} \rightarrow \mathcal{H}_{1}$ from a Hilbert space $\mathcal{H}_{0}$ to a Hilbert space $\mathcal{H}_{1}$ is a linear manifold in the Hilbert space $\mathcal{H}_{0} \oplus \mathcal{H}_{1}$. If $\mathcal{H}_{0}=\mathcal{H}_{1}=: \mathcal{H}$ one speaks of a linear relation $T$ in $\mathcal{H}$. The set of all closed linear relations from $\mathcal{H}_{0}$ to $\mathcal{H}_{1}$ (in $\mathcal{H}$ ) will be denoted by $\widetilde{\mathcal{C}}\left(\mathcal{H}_{0}, \mathcal{H}_{1}\right)$ $(\widetilde{\mathcal{C}}(\mathcal{H}))$. A closed linear operator $T$ from $\mathcal{H}_{0}$ to $\mathcal{H}_{1}$ is identified with its graph $\operatorname{gr} T \in \widetilde{\mathcal{C}}\left(\mathcal{H}_{0}, \mathcal{H}_{1}\right)$.

For a linear relation $T \in \widetilde{\mathcal{C}}\left(\mathcal{H}_{0}, \mathcal{H}_{1}\right)$ we denote by $\operatorname{dom} T, \operatorname{ran} T, \operatorname{ker} T$ and mul $T$ the domain, range, kernel and the multivalued part of $T$ respectively. For $T \in \widetilde{\mathcal{C}}\left(\mathcal{H}_{0}, \mathcal{H}_{1}\right)$ we will denote by $T^{-1}\left(\in \widetilde{\mathcal{C}}\left(\mathcal{H}_{1}, \mathcal{H}_{0}\right)\right)$ and $T^{*}\left(\in \widetilde{\mathcal{C}}\left(\mathcal{H}_{1}, \mathcal{H}_{0}\right)\right)$ the inverse and adjoint linear relations of $T$ respectively.

\subsection{The class $R[\mathcal{H}]$ of Nevanlinna operator functions}

Recall that an operator function $\Phi(\cdot): \mathbb{C}_{+} \rightarrow[\mathcal{H}]$ is called a Nevanlinna function (and is referred to the class $R[\mathcal{H}]$ ) if it is holomorphic and $\operatorname{Im} \Phi(\lambda) \geq 0, \quad \lambda \in \mathbb{C}_{+}$. We denote by $R_{u}[\mathcal{H}]$ the class of all functions $\Phi(\cdot) \in R[\mathcal{H}]$ such that the operator $\operatorname{Im} \Phi(\lambda)$ is invertible for all $\lambda \in \mathbb{C}_{+}$.

The following lemma will be useful in the sequel. 
Lemma 2.1. Let $M(\cdot) \in R_{u}[\mathcal{H}]$, let $\lambda_{0} \in \mathbb{C}_{+}$and let

$$
C(\lambda)=\left(M(\lambda)-\lambda_{0}\right)\left(M(\lambda)+\lambda_{0}\right)^{-1}, \quad \lambda \in \mathbb{C}_{+} .
$$

Then $\|C(\lambda)\| \leq 1, \lambda \in \mathbb{C}_{+}$, and the equality

$$
\lim _{y \rightarrow+\infty} y(\|h\|-\|C(i y) h\|)=+\infty, \quad h \in \mathcal{H}, \quad h \neq 0
$$

holds if and only if

$$
\lim _{y \rightarrow+\infty} \frac{1}{i y} M(i y)=0 \text { and } \lim _{y \rightarrow+\infty} y \cdot \operatorname{Im}(M(i y) h, h)=+\infty, h \in \mathcal{H}, h \neq 0 .
$$

Proof. According to [12] there exists a Hilbert space $\mathfrak{H}$, a symmetric operator $A$ in $\mathfrak{H}$ and a boundary triplet for $A^{*}$ such that $M(\cdot)$ is the Weyl function of this triplet. Moreover, the operator $A$ is densely defined if and only if (2.3) holds. Next, according to [28] $C(\lambda)$ is the characteristic function of $A$ in the sense of [38] and by Theorem 3.3 in [38] $A$ is densely defined if and only if (2.2) holds. Thus both the conditions (2.2) and (2.3) are equivalent to the dense definiteness of $A$ and hence (2.2) is equivalent to (2.3).

2.3. The classes $\widetilde{R}\left(\mathcal{H}_{0}, \mathcal{H}_{1}\right), \widetilde{R}_{\mathcal{H}_{1}}\left(\mathcal{H}_{0}\right)$ and $\widetilde{R}(\mathcal{H})$

In the following $\mathcal{H}_{0}$ is a Hilbert space, $\mathcal{H}_{1}$ is a subspace in $\mathcal{H}_{0}, \mathcal{H}_{2}:=$ $\mathcal{H}_{0} \ominus \mathcal{H}_{1}, P_{1}:=P_{\mathcal{H}_{0}, \mathcal{H}_{1}}$ and $P_{2}=P_{\mathcal{H}_{2}}$. Clearly,

$$
\mathcal{H}_{0}=\mathcal{H}_{1} \oplus \mathcal{H}_{2}=\mathcal{H}_{2} \oplus \mathcal{H}_{1} .
$$

Definition 2.2. [31] A relation $\theta \in \widetilde{\mathcal{C}}\left(\mathcal{H}_{0}, \mathcal{H}_{1}\right)$ belongs to the class $A c\left(\mathcal{H}_{0}, \mathcal{H}_{1}\right)$ if

$2 \operatorname{Im}\left(h_{1}, h_{0}\right)_{\mathcal{H}_{0}}+\left\|P_{2} h_{0}\right\|^{2} \leq 0,\left\{h_{0}, h_{1}\right\} \in \theta$, and $\left(\theta+\lambda P_{1}\right)^{-1} \in\left[\mathcal{H}_{1}, \mathcal{H}_{0}\right]$ for some (and hence for all) $\lambda \in \mathbb{C}_{-}$.

A function $\tau(\cdot): \mathbb{C}_{+} \rightarrow \widetilde{\mathcal{C}}\left(\mathcal{H}_{0}, \mathcal{H}_{1}\right)$ is referred to the class $\widetilde{R}\left(\mathcal{H}_{0}, \mathcal{H}_{1}\right)$ if $-\tau(\lambda) \in A c\left(\mathcal{H}_{0}, \mathcal{H}_{1}\right), \lambda \in \mathbb{C}_{+}$, and the operator-function $\left(\tau(\lambda)+i P_{1}\right)^{-1}$ is holomorphic on $\mathbb{C}_{+}$.

Let $\mathcal{K}$ be a Hilbert space. For a function $\tau(\cdot): \mathbb{C}_{+} \rightarrow \widetilde{\mathcal{C}}\left(\mathcal{H}_{0}, \mathcal{H}_{1}\right)$ and a pair of operator functions $C_{j}(\cdot): \mathbb{C}_{+} \rightarrow\left[\mathcal{H}_{j}, \mathcal{K}\right], j \in\{0,1\}$, we write $\tau(\lambda)=\left\{C_{0}(\lambda), C_{1}(\lambda)\right\}$ if

$$
\tau(\lambda)=\left\{\left\{h_{0}, h_{1}\right\} \in \mathcal{H}_{0} \oplus \mathcal{H}_{1}: C_{0}(\lambda) h_{0}+C_{1}(\lambda) h_{1}=0\right\}, \quad \lambda \in \mathbb{C}_{+}
$$


Proposition 2.3. [31] The equality $\tau(\lambda)=\left\{C_{0}(\lambda), C_{1}(\lambda)\right\}$ establishes a bijective correspondence between all functions $\tau(\cdot) \in \widetilde{R}\left(\mathcal{H}_{0}, \mathcal{H}_{1}\right)$ and all pairs of holomorphic operator-functions $C_{j}(\cdot): \mathbb{C}_{+} \rightarrow\left[\mathcal{H}_{j}, \mathcal{H}_{0}\right], j \in$ $\{0,1\}$, satisfying

$$
2 \operatorname{Im}\left(C_{1}(\lambda) C_{01}^{*}(\lambda)\right)+C_{02}(\lambda) C_{02}^{*}(\lambda) \geq 0, \quad\left(C_{0}(\lambda)-i C_{1}(\lambda) P_{1}\right)^{-1} \in\left[\mathcal{H}_{0}\right] .
$$

for all $\lambda \in \mathbb{C}_{+}$. Here $C_{0 j}(\cdot), j \in\{0,1\}$, are the entries of the block representation

$$
C_{0}(\lambda)=\left(C_{01}(\lambda), C_{02}(\lambda)\right): \mathcal{H}_{1} \oplus \mathcal{H}_{2} \rightarrow \mathcal{H}_{0}
$$

Proposition 2.3 enables one to identify a function $\tau(\cdot) \in \widetilde{R}\left(\mathcal{H}_{0}, \mathcal{H}_{1}\right)$ and the corresponding pair $\left\{C_{0}(\cdot), C_{1}(\cdot)\right\}$ (more precisely the equivalence class of such pairs [31]).

If $\mathcal{H}_{1}=\mathcal{H}_{0}=: \mathcal{H}$, then the class $\widetilde{R}(\mathcal{H}, \mathcal{H})$ coincides with the wellknown class $\widetilde{R}(\mathcal{H})$ of Nevanlinna $\widetilde{\mathcal{C}}(\mathcal{H})$-valued functions (Nevanlinna operator pairs) $\tau(\lambda)=\left\{C_{0}(\lambda), C_{1}(\lambda)\right\}$ with $C_{j}(\cdot): \mathbb{C}_{+} \rightarrow[\mathcal{H}]$. In this case the class $\widetilde{R}^{0}(\mathcal{H})$ is defined as the set of all $\tau(\cdot) \in \widetilde{R}(\mathcal{H})$ such that $\tau(\lambda) \equiv$ $\theta=\left\{C_{0}, C_{1}\right\}, \quad \lambda \in \mathbb{C}_{+}$, with $\theta=\theta^{*} \in \widetilde{\mathcal{C}}(\mathcal{H})$. Clearly, $R[\mathcal{H}] \subset \widetilde{R}(\mathcal{H})$.

Definition 2.4. A function (operator pair) $\widetilde{\tau}(\lambda)=\left\{\widetilde{C}_{0}(\lambda), \widetilde{C}_{1}(\lambda)\right\} \in$ $\widetilde{R}\left(\mathcal{H}_{0}\right)$ will be referred to the class $\widetilde{R}_{\mathcal{H}_{1}}\left(\mathcal{H}_{0}\right)$ if

$$
\widetilde{\tau}(\lambda) \cap \mathcal{H}_{2}^{2}=\left\{\left\{h_{2}, \frac{i}{2} h_{2}\right\}: h_{2} \in \mathcal{H}_{2}\right\}\left(=\operatorname{gr}\left(\frac{i}{2} I_{\mathcal{H}_{2}}\right)\right), \quad \lambda \in \mathbb{C}_{+} .
$$

Clearly, in the case $\mathcal{H}_{0}=\mathcal{H}_{1}=: \mathcal{H}$ one has $\widetilde{R}_{\mathcal{H}}(\mathcal{H})=\widetilde{R}(\mathcal{H})$.

The following lemma is obvious.

Lemma 2.5. (1) The operator pair $\widetilde{\tau}(\lambda)=\left\{\widetilde{C}_{0}(\lambda), \widetilde{C}_{1}(\lambda)\right\} \in \widetilde{R}\left(\mathcal{H}_{0}\right)$ belongs to $\widetilde{R}_{\mathcal{H}_{1}}\left(\mathcal{H}_{0}\right)$ if and only if the operator functions $\widetilde{C}_{j}(\lambda)$ admit the representation

$$
\begin{gathered}
\widetilde{C}_{0}(\lambda)=\left(C_{01}(\lambda), \frac{1}{2} C_{02}(\lambda)\right): \mathcal{H}_{1} \oplus \mathcal{H}_{2} \rightarrow \mathcal{H}_{0}, \\
\widetilde{C}_{1}(\lambda)=\left(C_{1}(\lambda), i C_{02}(\lambda)\right): \mathcal{H}_{1} \oplus \mathcal{H}_{2} \rightarrow \mathcal{H}_{0}, \quad \lambda \in \mathbb{C}_{+}
\end{gathered}
$$

with certain operator functions $C_{01}(\lambda), C_{02}(\lambda)$ and $C_{1}(\lambda)$. The operatorfunction $B(\cdot) \in R\left[\mathcal{H}_{0}\right]$ belongs to $\widetilde{R}_{\mathcal{H}_{1}}\left(\mathcal{H}_{0}\right)$ if and only if it admits the block representation

$$
B(\lambda)=\left(\begin{array}{cc}
B_{1}(\lambda) & 0 \\
B_{2}(\lambda) & \frac{i}{2} I_{\mathcal{H}_{2}}
\end{array}\right): \mathcal{H}_{1} \oplus \mathcal{H}_{2} \rightarrow \mathcal{H}_{1} \oplus \mathcal{H}_{2}, \quad \lambda \in \mathbb{C}_{+} .
$$

(2) The equalities (2.6) and (2.7) establish a bijective correspondence between all pairs $\tau(\lambda)=\left\{C_{0}(\lambda), C_{1}(\lambda)\right\} \in \widetilde{R}\left(\mathcal{H}_{0}, \mathcal{H}_{1}\right)$ with $C_{0}(\lambda)$ of the form (2.5) and all pairs $\widetilde{\tau}(\lambda)=\left\{\widetilde{C}_{0}(\lambda), \widetilde{C}_{1}(\lambda)\right\} \in \widetilde{R}_{\mathcal{H}_{1}}\left(\mathcal{H}_{0}\right)$. 
In the following proposition we give a parametrization of the class $\widetilde{R}_{\mathcal{H}_{1}}\left(\mathcal{H}_{0}\right)$ in terms of contractive operator-functions.

Proposition 2.6. The equalities

$$
\begin{aligned}
& \widetilde{C}_{0}(\lambda)=\left(\begin{array}{cc}
\frac{1}{\sqrt{2}} I_{\mathcal{H}_{2}} & i K_{2}(\lambda) \\
0 & i\left(K_{1}(\lambda)+I_{\mathcal{H}_{1}}\right)
\end{array}\right): \mathcal{H}_{2} \oplus \mathcal{H}_{1} \rightarrow \mathcal{H}_{2} \oplus \mathcal{H}_{1} \\
& \widetilde{C}_{1}(\lambda)=\left(\begin{array}{cc}
i \sqrt{2} I_{\mathcal{H}_{2}} & K_{2}(\lambda) \\
0 & K_{1}(\lambda)-I_{\mathcal{H}_{1}}
\end{array}\right): \mathcal{H}_{2} \oplus \mathcal{H}_{1} \rightarrow \mathcal{H}_{2} \oplus \mathcal{H}_{1}
\end{aligned}
$$

give a bijective correspondence between all operator-functions $\left(K_{1}(\lambda), K_{2}(\lambda)\right)^{\top} \quad$ belonging $\quad$ to $\quad \mathbf{C}\left[\mathcal{H}_{1}, \mathcal{H}_{1} \oplus \mathcal{H}_{2}\right] \quad$ and $\quad$ all pairs $\widetilde{\tau}(\lambda)=\left\{\widetilde{C}_{0}(\lambda), \widetilde{C}_{1}(\lambda)\right\} \in \widetilde{R}_{\mathcal{H}_{1}}\left(\mathcal{H}_{0}\right)$.

Proof. One can easily verify that the equality $X\left\{f_{0}, f_{1}\right\}=\left\{h_{0}, h_{1}\right\}$ with

$$
\begin{gathered}
P_{1} h_{0}=\frac{1}{\sqrt{2}}\left(f^{\prime}+i P_{1} f_{0}\right), \quad P_{2} h_{0}=P_{2} f_{0}, \\
h_{1}=\frac{1}{\sqrt{2}}\left(f^{\prime}-i P_{1} f_{0}\right), \quad\left\{f_{0}, f_{1}\right\} \in \mathcal{H}_{0} \oplus \mathcal{H}_{1}
\end{gathered}
$$

defines an automorphism $X \in\left[\mathcal{H}_{0} \oplus \mathcal{H}_{1}\right]$ such that $\left\|h_{0}\right\|^{2}-\left\|h_{1}\right\|^{2}=$ $2 \operatorname{Im}\left(f_{1}, f_{0}\right)_{\mathcal{H}_{0}}+\left\|P_{2} f_{0}\right\|^{2}$. Therefore the equalities

$$
\begin{gathered}
K_{1}(\lambda)=I_{\mathcal{H}_{1}}-2 i P_{1}\left(\tau(\lambda)+i P_{1}\right)^{-1}, \\
K_{2}(\lambda)=-\sqrt{2} P_{2}\left(\tau(\lambda)+i P_{1}\right)^{-1}, \quad \lambda \in \mathbb{C}_{+}
\end{gathered}
$$

establish a bijective correspondence between all $\tau(\lambda) \in \widetilde{R}\left(\mathcal{H}_{0}, \mathcal{H}_{1}\right)$ and all operator-functions $\left(K_{1}(\lambda), K_{2}(\lambda)\right)^{\top} \in \mathbf{C}\left[\mathcal{H}_{1}, \mathcal{H}_{1} \oplus \mathcal{H}_{2}\right]$. It follows from (2.3) that $\left\{f_{0}, f_{1}\right\} \in \tau(\lambda)$ if and only if

$$
\begin{gathered}
i\left(K_{1}(\lambda)+I_{\mathcal{H}_{1}}\right) P_{1} f_{0}+\left(K_{1}(\lambda)-I_{\mathcal{H}_{1}}\right) f_{1}=0, \\
i K_{2}(\lambda) P_{1} f_{0}+\sqrt{2} P_{2} f_{0}+K_{2}(\lambda) f_{1}=0 .
\end{gathered}
$$

Therefore the equalities

$$
\begin{gathered}
C_{0}(\lambda)=\left(\begin{array}{cc}
\sqrt{2} I_{\mathcal{H}_{2}} & i K_{2}(\lambda) \\
0 & i\left(K_{1}(\lambda)+I_{\mathcal{H}_{1}}\right)
\end{array}\right): \mathcal{H}_{2} \oplus \mathcal{H}_{1} \rightarrow \mathcal{H}_{2} \oplus \mathcal{H}_{1} \\
C_{1}(\lambda)=\left(K_{2}(\lambda), K_{1}(\lambda)-I_{\mathcal{H}_{1}}\right)^{\top}: \mathcal{H}_{1} \rightarrow \mathcal{H}_{2} \oplus \mathcal{H}_{1}
\end{gathered}
$$

gives a bijective correspondence between all functions $\left(K_{1}(\lambda), K_{2}(\lambda)\right)^{\top} \in$ $\mathbf{C}\left[\mathcal{H}_{1}, \mathcal{H}_{1} \oplus \mathcal{H}_{2}\right]$ and all functions $\tau(\lambda)=\left\{C_{0}(\lambda), C_{1}(\lambda)\right\} \in \widetilde{R}\left(\mathcal{H}_{0}, \mathcal{H}_{1}\right)$. Now the statement of the proposition follows from Lemma 2.5, (2). 


\subsection{Boundary pairs for symmetric relations}

As is known a linear relation $A$ in $\mathfrak{H}$ is called symmetric (self-adjoint) if $A \subset A^{*}$ (resp. $A=A^{*}$ ). Assume that $A \in \widetilde{\mathcal{C}}(\mathfrak{H})$ is a symmetric relation, $\mathfrak{N}_{\lambda}(A)=\operatorname{ker}\left(A^{*}-\lambda\right)(\lambda \in \mathbb{C})$ is a defect subspace of $A$ and $\widehat{\mathfrak{N}}_{\lambda}(A)=\left\{\{f, \lambda f\}: f \in \mathfrak{N}_{\lambda}(A)\right\}$.

Recall that a pair $\left\{\mathcal{H}_{0} \oplus \mathcal{H}_{1}, \Gamma\right\}$ with a linear relation $\Gamma: \mathfrak{H} \oplus \mathfrak{H} \rightarrow$ $\mathcal{H}_{0} \oplus \mathcal{H}_{1}$ is called a boundary pair for $A^{*}$ if $\overline{\operatorname{dom} \Gamma}=A^{*}$, the abstract Green's identity

$$
\left(f^{\prime}, g\right)_{\mathfrak{H}}-\left(f, g^{\prime}\right)_{\mathfrak{H}}=\left(h_{1}, x_{0}\right)_{\mathcal{H}_{0}}-\left(h_{0}, x_{1}\right)_{\mathcal{H}_{0}}+i\left(P_{2} h_{0}, P_{2} x_{0}\right)_{\mathcal{H}_{0}}
$$

holds for every $\left\{f \oplus f^{\prime}, h_{0} \oplus h_{1}\right\},\left\{g \oplus g^{\prime}, x_{0} \oplus x_{1}\right\} \in \Gamma$ and a certain maximality condition is satisfied [10,30].

The following proposition is immediate from [30, Section 3].

Proposition 2.7. Let $\left\{\mathcal{H}_{0} \oplus \mathcal{H}_{1}, \Gamma\right\}$ be a boundary pair for $A^{*}$ with $\operatorname{dim} \mathcal{H}_{0}<\infty$, let $\Gamma_{0}: \mathfrak{H} \oplus \mathfrak{H} \rightarrow \mathcal{H}_{0}$ be the linear relations, given by $\Gamma_{0}=P_{\mathcal{H}_{0} \oplus\{0\}} \Gamma$ and let

$$
\mathcal{K}_{\Gamma}:=\operatorname{mul}(\operatorname{mul} \Gamma)=\left\{h_{1} \in \mathcal{H}_{1}:\left\{0 \oplus 0,0 \oplus h_{1}\right\} \in \Gamma\right\}, \quad \mathcal{K}_{\Gamma} \subset \mathcal{H}_{1} .
$$

Assume also that $\pi_{1}$ is the orthoprojection in $\mathfrak{H} \oplus \mathfrak{H}$ onto $\mathfrak{H} \oplus\{0\}$. If $\mathcal{K}_{\Gamma}=\{0\}$, then the equalities

$$
\begin{gathered}
\gamma_{+}(\lambda)=\pi_{1}\left(\Gamma_{0}\left\lceil\widehat{\mathfrak{N}}_{\lambda}(A)\right)^{-1}, \quad \lambda \in \mathbb{C}_{+}\right. \\
\operatorname{gr} M_{+}(\lambda)=\left\{h_{0} \oplus h_{1}:\left\{f \oplus \lambda f, h_{0} \oplus h_{1}\right\} \in \Gamma\right. \\
\text { with some } \left.f \in \mathfrak{N}_{\lambda}(A)\right\}, \quad \lambda \in \mathbb{C}_{+}
\end{gathered}
$$

correctly define the holomorphic operator function $\gamma_{+}(\cdot): \mathbb{C}_{+} \rightarrow\left[\mathcal{H}_{0}, \mathfrak{H}\right]$ (the $\gamma$-field) and $M_{+}(\cdot): \mathbb{C}_{+} \rightarrow\left[\mathcal{H}_{0}, \mathcal{H}_{1}\right]$ (the Weyl function of the pair $\left.\left\{\mathcal{H}_{0} \oplus \mathcal{H}_{1}, \Gamma\right\}\right)$. Moreover, if

$$
M_{+}(\lambda)=\left(M(\lambda), N_{+}(\lambda)\right): \mathcal{H}_{1} \oplus \mathcal{H}_{2} \rightarrow \mathcal{H}_{1}, \quad \lambda \in \mathbb{C}_{+}
$$

is the block representation of $M_{+}(\cdot)$, then the equality

$$
\mathcal{M}(\lambda)=\left(\begin{array}{cc}
M(\lambda) & N_{+}(\lambda) \\
0 & \frac{i}{2} I_{\mathcal{H}_{2}}
\end{array}\right): \underbrace{\mathcal{H}_{1} \oplus \mathcal{H}_{2}}_{\mathcal{H}_{0}} \rightarrow \underbrace{\mathcal{H}_{1} \oplus \mathcal{H}_{2}}_{\mathcal{H}_{0}}, \quad \lambda \in \mathbb{C}_{+}
$$

defines the operator-function $\mathcal{M}(\cdot) \in R_{u}\left[\mathcal{H}_{0}\right]$ and the following identity holds

$$
\mathcal{M}(\mu)-\mathcal{M}^{*}(\lambda)=(\mu-\bar{\lambda}) \gamma_{+}^{*}(\lambda) \gamma_{+}(\mu), \quad \mu, \lambda \in \mathbb{C}_{+} .
$$

Remark 2.8. A boundary pair for $A^{*}$ and its Weyl function is a useful generalization of the well known concept of a boundary triplet for $A^{*}$ $[8,19,29]$ and its Weyl function $[11,28,29]$. 


\section{Pseudospectral and spectral functions of symmetric systems}

\subsection{Notations}

For an interval $\mathcal{I}=[a, b\rangle \subset \mathbb{R}$ and a finite-dimensional Hilbert space $\mathbb{H}$ we denote by $A C(\mathcal{I} ; \mathbb{H})$ the set of all functions $f(\cdot): \mathcal{I} \rightarrow \mathbb{H}$, which are absolutely continuous on each segment $[\alpha, \beta] \subset \mathcal{I}$.

Assume that $\Delta(\cdot): \mathcal{I} \rightarrow[\mathbb{H}]$ is a locally integrable function such that $\Delta(t) \geq 0$ a.e. on $\mathcal{I}$. Denote by $\mathcal{L}_{\Delta}^{2}(\mathcal{I} ; \mathbb{H})$ the semi-Hilbert space of Borel measurable functions $f(\cdot): \mathcal{I} \rightarrow \mathbb{H}$ satisfying $\int_{\mathcal{I}}(\Delta(t) f(t), f(t))_{\mathbb{H}} d t<\infty$ (see e.g. [15, Chapter 13.5]), by $L_{\Delta}^{2}(\mathcal{I} ; \mathbb{H})$ the Hilbert space of equivalence classes in $\mathcal{L}_{\Delta}^{2}(\mathcal{I} ; \mathbb{H})$ with respect to the semi-norm in $\mathcal{L}_{\Delta}^{2}(\mathcal{I} ; \mathbb{H})$ and by $\pi_{\Delta}$ the quotient map from $\mathcal{L}_{\Delta}^{2}(\mathcal{I} ; \mathbb{H})$ onto $L_{\Delta}^{2}(\mathcal{I} ; \mathbb{H})$.

For a finite-dimensional Hilbert space $\mathcal{K}$ we denote by $\mathcal{L}_{\Delta}^{2}[\mathcal{K}, \mathbb{H}]$ the set of all Borel measurable operator-functions $F(\cdot): \mathcal{I} \rightarrow[\mathcal{K}, \mathbb{H}]$ such that $F(t) h \in \mathcal{L}_{\Delta}^{2}(\mathcal{I} ; \mathbb{H}), h \in \mathcal{K}$. Moreover, we let $\mathcal{L}_{\Delta}^{2}[\mathbb{H}]:=\mathcal{L}_{\Delta}^{2}[\mathbb{H}, \mathbb{H}]$

In the following for an operator-valued distribution function $\sigma(\cdot)$ : $\mathbb{R} \rightarrow[\mathbb{H}]$ we denote by $\mathcal{L}^{2}(\sigma ; \mathbb{H})$ the semi-Hilbert space of Borel-measurable functions $g(\cdot): \mathbb{R} \rightarrow \mathbb{H}$ such that $\int_{\mathbb{R}}(d \sigma(s) g(s), g(s))<\infty$ and by $L^{2}(\sigma ; \mathbb{H})$ the Hilbert space of all equivalence classes in $\mathcal{L}^{2}(\sigma ; \mathbb{H})$ with respect to the seminorm $\|\cdot\|_{\mathcal{L}^{2}(\sigma ; \mathbb{H})}$ (see e.g. [15, Chapter 13.5]). Moreover, we denote by $\pi_{\sigma}$ the quotient map from $\mathcal{L}^{2}(\sigma ; \mathbb{H})$ onto $L^{2}(\sigma ; \mathbb{H})$.

\subsection{Symmetric systems}

Let $H$ and $\widehat{H}$ be finite dimensional Hilbert spaces and let

$$
\mathbb{H}:=H \oplus \widehat{H} \oplus H, \quad n:=\operatorname{dim} \mathbb{H} .
$$

A first order symmetric system of differential equations on an interval $\mathcal{I}=[a, b\rangle,-\infty<a<b \leq \infty$, (with the regular endpoint $a$ ) is of the form

$$
J y^{\prime}-A(t) y=\lambda \Delta(t) y, \quad t \in \mathcal{I}, \quad \lambda \in \mathbb{C},
$$

where $J$ is the operator $(1.2)$ and $A(\cdot)$ and $\Delta(\cdot)$ are [HH]-valued functions on $\mathcal{I}$ integrable on each compact interval $[a, \beta] \subset \mathcal{I}$ and such that $A(t)=$ $A^{*}(t)$ and $\Delta(t) \geq 0$ (a.e. on $\mathcal{I}$ ). In the case $A(t) \equiv 0$ system (3.1) is called canonical.

A function $y \in A C(\mathcal{I} ; \mathbb{H})$ is a solution of system (3.1) if equality (3.1) holds a.e. on $\mathcal{I}$. An operator function $Y(\cdot, \lambda): \mathcal{I} \rightarrow[\mathcal{K}, \mathbb{H}]$ is an operator solution of (3.1) if $y(t)=Y(t, \lambda) h$ is a solution of (3.1) for every $h \in \mathcal{K}$ (here $\mathcal{K}$ is a Hilbert space with $\operatorname{dim} \mathcal{K}<\infty$ ). 
Let $\mathcal{N}_{\lambda}, \lambda \in \mathbb{C}$, be the linear space of all solutions of the system (3.1) belonging to $\mathcal{L}_{\Delta}^{2}(\mathcal{I} ; \mathbb{H})$. According to $[22,27]$ the numbers $N_{ \pm}=$ $\operatorname{dim} \mathcal{N}_{\lambda}, \lambda \in \mathbb{C}_{ \pm}$, do not depend on $\lambda$ in either $\mathbb{C}_{+}$or $\mathbb{C}_{-}$. These numbers are called the formal deficiency indices of the system [22]. Clearly $N_{ \pm} \leq$ $n$.

In the following for an operator solution $Y(\cdot, \lambda) \in \mathcal{L}_{\Delta}^{2}[\mathcal{K}, \mathbb{H}]$ of $(3.1)$ we denote by $Y(\lambda)$ the linear operator from $\mathcal{K}$ to $\mathcal{N}_{\lambda}$ given by

$$
(Y(\lambda) h)(t)=Y(t, \lambda) h, \quad h \in \mathcal{K} .
$$

For any $\lambda \in \mathbb{C}$ the space $\mathcal{N}_{\lambda}^{\prime}$ of all solutions $y$ of (3.1) with $\Delta(t) y(t)=$ 0 (a.e. on $\mathcal{I}$ ) is a subspace of $\mathcal{N}_{\lambda}$; moreover, $\mathcal{N}_{\lambda}^{\prime}$ does not depend on $\lambda$. The space $\mathcal{N}=\mathcal{N}_{\lambda}^{\prime}, \lambda \in \mathbb{C}$, is called the null manifold of the system [22].

As is known $[7,20,27,30]$ system (3.1) gives rise to the maximal linear relations $\mathcal{T}_{\text {max }}$ and the minimal relation $\mathcal{T}_{\min }$ in $\mathcal{L}_{\Delta}^{2}(\mathcal{I} ; \mathbb{H})$. Moreover, the equalities $T_{\max }=\left(\pi_{\Delta} \oplus \pi_{\Delta}\right) \mathcal{T}_{\max }$ and $T_{\min }=\left(\pi_{\Delta} \oplus \pi_{\Delta}\right) \mathcal{T}_{\min }$ define the maximal and minimal relations $T_{\max }$ and $T_{\min }$ in $L_{\Delta}^{2}(\mathcal{I} ; \mathbb{H})$ respectively. It turns out that $T_{\min }$ is a closed symmetric linear relation in $L_{\Delta}^{2}(\mathcal{I} ; \mathbb{H})$ and $T_{\min }^{*}=T_{\max }$. Observe also that the Lagrange's identity

$$
(f, z)_{\Delta}-(y, g)_{\Delta}=[y, z]_{b}-(J y(a), z(a)), \quad\{y, f\},\{z, g\} \in \mathcal{T}_{\max }
$$

holds with $[y, z]_{b}:=\lim _{t \rightarrow b}(J y(t), z(t)), y, z \in \operatorname{dom} \mathcal{T}_{\max }$.

With each subspace $\theta \subset \mathbb{H}$ we associate the subspace $\theta^{\times} \subset \mathbb{H}$ given by

$$
\theta^{\times}=\mathbb{H} \ominus J \theta=\{h \in \mathbb{H}:(J h, k)=0, k \in \theta\} .
$$

Moreover, we denote by $\operatorname{Sym}(\mathbb{H})$ the set of all subspaces $\theta$ in $\mathbb{H}$ satisfying $\theta \subset \theta^{\times}$or, equivalently, $(J h, k)=0, h, k \in \theta$.

\subsection{Pseudospectral and spectral functions}

In what follows we put $\mathfrak{H}:=L_{\Delta}^{2}(\mathcal{I} ; \mathbb{H})$ and denote by $\mathfrak{H}_{b}$ the set of all $\widetilde{f} \in \mathfrak{H}$ with the following property: there exists $\beta_{\widetilde{f}} \in \mathcal{I}$ such that for some (and hence for all) function $f \in \tilde{f}$ the equality $\Delta(t) f(t)=0$ holds a.e. on $\left(\beta_{\widetilde{f}}, b\right)$.

With each subspace $\theta \in \mathbb{H}$ one associates a linear relation $T_{\theta \times} \in \widetilde{\mathcal{C}}(\mathfrak{H})$ given by

$$
\begin{gathered}
T_{\theta^{\times}}=\left\{\left\{\pi_{\Delta} y, \pi_{\Delta} f\right\}:\{y, f\} \in \mathcal{T}_{\max }, y(a) \in \theta^{\times}\right. \\
\text {and } \left.[y, z]_{b}=0, z \in \operatorname{dom} \mathcal{T}_{\max }\right\}
\end{gathered}
$$

The following assertion is obvious. 
Assertion 3.1. The multivalued part mul $T_{\theta^{\times}}$of $T_{\theta^{\times}}$is the set of all $\widetilde{f} \in \mathfrak{H}$ such that for some (and hence for all) $f(\cdot) \in \widetilde{f}$ there exists a solution $y$ of the system

$$
J y^{\prime}-A(t) y=\Delta(t) f(t), \quad t \in \mathcal{I}
$$

satisfying the relations

$$
\Delta(t) y(t)=0(\text { a.e. on } \mathcal{I}), \quad y(a) \in \theta^{\times} \text {and }[y, z]_{b}=0, z \in \operatorname{dom} \mathcal{T}_{\max } .
$$

Let $\theta$ be a subspace in $\mathbb{H}$. Moreover, let the following assumption (A0) be satisfied:

(A0) $\mathbb{H}_{0}^{\prime}$ is a subspace in $\mathbb{H}, K=K_{\theta} \in\left[\mathbb{H}_{0}^{\prime}, \mathbb{H}\right]$ is an operator such that ker $K_{\theta}=\{0\}$ and $K_{\theta} \mathbb{H}_{0}^{\prime}=\theta$ and $Y_{K}(\cdot, \lambda)\left(\in\left[\mathbb{H}_{0}^{\prime}, \mathbb{H}\right]\right)$ is an operator solution of (3.1) with $Y_{K}(a, \lambda)=K, \lambda \in \mathbb{C}$.

With each $\widetilde{f} \in \mathfrak{H}_{b}$ one associates the function $\widehat{f}(\cdot): \mathbb{R} \rightarrow \mathbb{H}_{0}^{\prime}$ given by

$$
\widehat{f}(s)=\int_{\mathcal{I}} Y_{K}^{*}(t, s) \Delta(t) f(t) d t, \quad f(\cdot) \in \widetilde{f} .
$$

Definition 3.2. [35] A distribution function $\sigma(\cdot): \mathbb{R} \rightarrow\left[\mathbb{H}_{0}^{\prime}\right]$ is called a pseudospectral function of the system (3.1) (with respect to the operator $\left.K=K_{\theta}\right)$ if $\widehat{f} \in \mathcal{L}^{2}\left(\sigma ; \mathbb{H}_{0}^{\prime}\right)$ for all $\widetilde{f} \in \mathfrak{H}_{b}$ and the operator $V \widetilde{f}:=\pi_{\sigma} \widehat{f}, \widetilde{f} \in$ $\mathfrak{H}_{b}$, admits a continuation to a partial isometry $V_{\sigma} \in\left[\mathfrak{H}, L^{2}\left(\sigma ; \mathbb{H}_{0}^{\prime}\right)\right]$ with $\operatorname{ker} V_{\sigma}=\operatorname{mul} T_{\theta^{\times}}$.

If $V$ admits a continuation to an isometry $V_{\sigma} \in\left[\mathfrak{H}, L^{2}\left(\sigma ; \mathbb{H}_{0}^{\prime}\right)\right]$, then $\sigma(\cdot)$ is called a spectral function.

The operator $V_{\sigma}$ is called the (generalized) Fourier operator corresponding to $\sigma(\cdot)$.

Remark 3.3. (1) Definition 3.2 is motivated by the fact that a pseudospectral function possesses a useful extremal property (for more details see [35]).

(2) It follows from [35, Proposition 3.12] that a pseudospectral (in particular spectral) function $\sigma(\cdot)$ with respect to the operator $K_{\theta} \in\left[\mathbb{H}_{0}^{\prime}, \mathbb{H}\right]$ is uniquely characterized by the subspace $\theta$ and does not depend in fact on a choice of $\mathbb{H}_{0}^{\prime}$ and $K_{\theta}$.

Proposition 3.4. [35] If mul $T_{\theta^{\times}} \neq\{0\}$, then the set of spectral functions is empty; otherwise the sets of spectral end pseudospectral functions (wit respect to $K_{\theta}$ ) coincide. 


\subsection{Decomposing boundary pairs}

Definition 3.5. [6,17] System (3.1) is called definite if $\mathcal{N}=\{0\}$ or, equivalently, if for some (and hence for all) $\lambda \in \mathbb{C}$ there exists only a trivial solution $y=0$ of this system satisfying $\Delta(t) y(t)=0$ (a.e. on $\mathcal{I}$ ).

Definition 3.6. [35] Let $\theta$ be a subspace in $\mathbb{H}$. System (3.1) is called $\theta$-definite if the conditions $y \in \mathcal{N}$ and $y(a) \in \theta$ yield $y=0$.

Remark 3.7. (1) Clearly, $\mathbb{H}$-definiteness is the same as definiteness.

(2) If system is definite, then obviously it is $\theta$-definite for any $\theta \in$ $\mathbb{H}$. Hence $\theta$-definiteness is generally speaking a weaker condition then definiteness.

(3) If ker $\Delta(t)=0$ (a.e. on $\mathcal{I}$ ), then obviously system (3.1) is definite and by Assertion $3.1 \mathrm{mul} T_{\min }=\operatorname{mul} T_{\max }=\{0\}$ (that is $T_{\min }$ is a densely defined operator). Therefore in this case for any $\theta$ the sets of spectral and pseudospectral functions (with respect to $K_{\theta}$ ) coincide.

Lemma 3.8. $[2,30]$ For any system (3.1) with $N_{-} \leq N_{+}$there exist a finite dimensional Hilbert space $\widetilde{\mathcal{H}}_{b}$, a subspace $\mathcal{H}_{b} \subset \widetilde{\mathcal{H}}_{b}$ and a surjective linear operator

$$
\Gamma_{b}^{\prime}=\left(\Gamma_{0 b}^{\prime}, \widehat{\Gamma}_{b}^{\prime}, \Gamma_{1 b}^{\prime}\right)^{\top}: \operatorname{dom} \mathcal{T}_{\max } \rightarrow \widetilde{\mathcal{H}}_{b} \oplus \widehat{H} \oplus \mathcal{H}_{b}
$$

such that for all $y, z \in \operatorname{dom} \mathcal{T}_{\max }$ the following identity is valid:

$[y, z]_{b}=\left(\Gamma_{0 b}^{\prime} y, \Gamma_{1 b}^{\prime} z\right)-\left(\Gamma_{1 b}^{\prime} y, \Gamma_{0 b}^{\prime} z\right)+i\left(P_{\mathcal{H}_{b}} \Gamma_{0 b}^{\prime} y, P_{\mathcal{H}_{b}} \Gamma_{0 b}^{\prime} z\right)+i\left(\widehat{\Gamma}_{b}^{\prime} y, \widehat{\Gamma}_{b}^{\prime} z\right)$

(here $\mathcal{H}_{b}^{\perp}=\widetilde{\mathcal{H}}_{b} \ominus \mathcal{H}_{b}$ ). Moreover,

$$
\operatorname{dim} \widetilde{\mathcal{H}}_{b}=N_{+}-\operatorname{dim} H-\operatorname{dim} \widehat{H}, \quad \operatorname{dim} \mathcal{H}_{b}=N_{-}-\operatorname{dim} H-\operatorname{dim} \widehat{H}
$$

Note that $\Gamma_{b}^{\prime} y$ is a singular boundary value of a function $y \in \operatorname{dom} \mathcal{T}_{\max }$ at the end point $b$ (for more details see [2, Remark 3.5]).

Below within this section we suppose the following assumptions:

(A1) $\theta$ is a subspace in $\mathbb{H}$ and $\theta^{\times} \in \operatorname{Sym}(\mathbb{H})$. Moreover, system (3.1) is $\theta$-definite and satisfies $N_{-} \leq N_{+}$.

(A2) $H_{1}$ is a subspace in $H, H_{1}^{\perp}=H \ominus H_{1}, \mathbb{H}_{0}$ is a subspace in $\mathbb{H}$ given by

$$
\mathbb{H}_{0}=H \oplus \widehat{H} \oplus H_{1}=H_{1}^{\perp} \oplus H_{1} \oplus \widehat{H} \oplus H_{1} .
$$

and $\widetilde{U} \in[\mathbb{H}]$ is an operator satisfying $\widetilde{U}^{*} J \widetilde{U}=J$ and $\widetilde{U} \mathbb{H}_{0}=\theta$ (existence of such an operator follows from [35, Lemma 3.1]).

(A3) $\widetilde{\mathcal{H}}_{b}$ and $\mathcal{H}_{b} \subset \widetilde{\mathcal{H}}_{b}$ are finite dimensional Hilbert spaces and $\Gamma_{b}^{\prime}$ is a surjective linear operator (3.8) satisfying (3.9) (see Lemma 3.8). 
Proposition 3.9. [30,35] Assume that $\Gamma_{a}: \operatorname{dom} \mathcal{T}_{\max } \rightarrow \mathbb{H}$ is the linear operator given by $\Gamma_{a} y=\widetilde{U}^{-1} y(a), y \in \operatorname{dom} \mathcal{T}_{\max }$, and

$$
\Gamma_{a}=\left(\Gamma_{0 a}^{1}, \Gamma_{0 a}^{2}, \widehat{\Gamma}_{a}, \Gamma_{1 a}^{2}, \Gamma_{1 a}^{1}\right)^{\top}: \operatorname{dom} \mathcal{T}_{\max } \rightarrow \underbrace{H_{1}^{\perp} \oplus H_{1}}_{H} \oplus \hat{H} \oplus \underbrace{H_{1} \oplus H_{1}^{\perp}}_{H}
$$

is the block representation of $\Gamma_{a}$. Moreover, let $\mathcal{H}_{0}$ and $\mathcal{H}_{1} \subset \mathcal{H}_{0}$ be finite dimensional Hilbert spaces and let $\Gamma_{j}^{\prime}: \operatorname{dom} \mathcal{T}_{\max } \rightarrow \mathcal{H}_{j}, j \in\{0,1\}$, be linear operators given by

$$
\begin{aligned}
& \mathcal{H}_{0}=H_{1}^{\perp} \oplus H_{1} \oplus \widehat{H} \oplus \widetilde{\mathcal{H}}_{b}, \quad \mathcal{H}_{1}=H_{1}^{\perp} \oplus H_{1} \oplus \widehat{H} \oplus \mathcal{H}_{b} \\
\Gamma_{0}^{\prime}= & \left(-\Gamma_{1 a}^{1},-\Gamma_{1 a}^{2}, i\left(\widehat{\Gamma}_{a}-\widehat{\Gamma}_{b}^{\prime}\right), \Gamma_{0 b}^{\prime}\right)^{\top}: \operatorname{dom} \mathcal{T}_{\max } \rightarrow H_{1}^{\perp} \oplus H_{1} \oplus \widehat{H} \oplus \widetilde{\mathcal{H}}_{b} \\
\Gamma_{1}^{\prime}= & \left(\Gamma_{0 a}^{1}, \Gamma_{0 a}^{2}, \frac{1}{2}\left(\widehat{\Gamma}_{a}+\widehat{\Gamma}_{b}^{\prime}\right),-\Gamma_{1 b}^{\prime}\right)^{\top}: \operatorname{dom} \mathcal{T}_{\max } \rightarrow H_{1}^{\perp} \oplus H_{1} \oplus \widehat{H} \oplus \mathcal{H}_{b} .
\end{aligned}
$$

Then a pair $\left\{\mathcal{H}_{0} \oplus \mathcal{H}_{1}, \Gamma\right\}$ with a linear relation $\Gamma: \mathfrak{H} \oplus \mathfrak{H} \rightarrow \mathcal{H}_{0} \oplus \mathcal{H}_{1}$ defined by

$$
\Gamma=\left\{\left\{\pi_{\Delta} y \oplus \pi_{\Delta} f, \Gamma_{0}^{\prime} y \oplus \Gamma_{1}^{\prime} y\right\}:\{y, f\} \in \mathcal{T}_{\max }\right\}
$$

is a boundary pair for $T_{\max }$ such that $\mathcal{K}_{\Gamma}=\{0\}$ (for $\mathcal{K}_{\Gamma}$ see (2.9)).

A boundary pair for $T_{\max }$ from Proposition 3.9 is called a decomposing boundary pair.

\subsection{Parametrization of pseudospectral and spectral functions}

Let $\left\{\mathcal{H}_{0} \oplus \mathcal{H}_{1}, \Gamma\right\}$ be the decomposing boundary pair for $T_{\max }$, let $M_{+}=M_{+}(\lambda), \lambda \in \mathbb{C}_{+}$, be the Weyl function of this pair and let

$$
\begin{aligned}
& M_{+}=\left(\begin{array}{llll}
M_{11} & M_{12} & M_{13} & M_{14} \\
M_{21} & M_{22} & M_{23} & M_{24} \\
M_{31} & M_{32} & M_{33} & M_{34} \\
M_{41} & M_{42} & M_{43} & M_{44}
\end{array}\right): \underbrace{H_{1}^{\perp} \oplus H_{1} \oplus \widehat{H} \oplus \widetilde{\mathcal{H}}_{b}}_{\mathcal{H}_{0}} \\
& \rightarrow \underbrace{H_{1}^{\perp} \oplus H_{1} \oplus \widehat{H} \oplus \mathcal{H}_{b}}_{\mathcal{H}_{1}},
\end{aligned}
$$

be the block representation of $M_{+}$with entries $M_{j k}=M_{j k}(\lambda)$. Note, that the operator functions $M_{j k}$ are defined explicitly in terms of the boundary values of respective operator solutions of (3.1) at the endpoints $a$ and $b$ (see [35, Proposition 4.13]). 
Next assume that $\dot{\mathcal{H}}_{0}$ and $\dot{\mathcal{H}}_{1} \subset \dot{\mathcal{H}}_{0}$ are finite dimensional Hilbert spaces given by

$$
\dot{\mathcal{H}}_{0}=H_{1} \oplus \widehat{H} \oplus \widetilde{\mathcal{H}}_{b}, \quad \dot{\mathcal{H}}_{1}=H_{1} \oplus \widehat{H} \oplus \mathcal{H}_{b}
$$

and let $\dot{\mathcal{H}}_{2}=\dot{\mathcal{H}}_{0} \ominus \dot{\mathcal{H}}_{1}$. Clearly $\dot{\mathcal{H}}_{2}=\mathcal{H}_{b}^{\perp}\left(=\widetilde{\mathcal{H}}_{b} \ominus \mathcal{H}_{b}\right)$ and hence

$$
\dot{\mathcal{H}}_{0}=\dot{\mathcal{H}}_{1} \oplus \mathcal{H}_{b}^{\perp} .
$$

Using the entries $M_{j k}=M_{j k}(\lambda)$ from (3.5) we introduce the holomorphic operator-functions $m_{0}=m_{0}(\lambda)\left(\in\left[\mathbb{H}_{0}\right]\right), S_{1}=S_{1}(\lambda)\left(\in\left[\dot{\mathcal{H}}_{0}, \mathbb{H}_{0}\right]\right), S_{2}=$ $S_{2}(\lambda)\left(\in\left[\mathbb{H}_{0}, \dot{\mathcal{H}}_{0}\right]\right)$ and $\dot{\mathcal{M}}=\dot{\mathcal{M}}(\lambda)\left(\in\left[\dot{\mathcal{H}}_{0}\right]\right), \lambda \in \mathbb{C}_{+}$, by setting

$$
\begin{aligned}
& m_{0}=\left(\begin{array}{cccc}
M_{11} & M_{12} & M_{13} & 0 \\
M_{21} & M_{22} & M_{23} & -\frac{1}{2} I_{H_{1}} \\
M_{31} & M_{32} & M_{33} & 0 \\
0 & -\frac{1}{2} I_{H_{1}} & 0 & 0
\end{array}\right): \underbrace{H_{1}^{\perp} \oplus H_{1} \oplus \widehat{H} \oplus H_{1}}_{\mathbb{H}_{0}} \\
& \rightarrow \underbrace{H_{1}^{\perp} \oplus H_{1} \oplus \widehat{H} \oplus H_{1}}_{\mathbb{H}_{0}} \\
& S_{1}=\left(\begin{array}{ccc}
M_{12} & M_{13} & M_{14} \\
M_{22} & M_{23} & M_{24} \\
M_{32} & M_{33}-\frac{i}{2} I_{\widehat{H}} & M_{34} \\
-I_{H_{1}} & 0 & 0
\end{array}\right): \underbrace{H_{1} \oplus \widehat{H} \oplus \widetilde{\mathcal{H}}_{b}}_{\dot{\mathcal{H}}_{0}} \\
& \rightarrow \underbrace{H_{1}^{\perp} \oplus H_{1} \oplus \widehat{H} \oplus H_{1}}_{\mathbb{H}_{0}} \\
& S_{2}=\left(\begin{array}{cccc}
M_{21} & M_{22} & M_{23} & -I_{H_{1}} \\
M_{31} & M_{32} & M_{33}+\frac{i}{2} I_{\widehat{H}} & 0 \\
M_{41} & M_{42} & M_{43} & 0
\end{array}\right): \underbrace{H_{1}^{\perp} \oplus H_{1} \oplus \widehat{H} \oplus H_{1}}_{\mathbb{H}_{0}} \\
& \rightarrow \underbrace{H_{1} \oplus \widehat{H} \oplus \widetilde{\mathcal{H}}_{b}}_{\dot{\mathcal{H}}_{0}} \\
& \dot{\mathcal{M}}=\left(\begin{array}{ccc}
M_{22} & M_{23} & M_{24} \\
M_{32} & M_{33} & M_{34} \\
M_{42} & M_{43} & M_{44}+\frac{i}{2} P_{\mathcal{H}_{b}^{\perp}}
\end{array}\right): \underbrace{H_{1} \oplus \widehat{H} \oplus \widetilde{\mathcal{H}}_{b}}_{\dot{\mathcal{H}}_{0}} \rightarrow \underbrace{H_{1} \oplus \widehat{H} \oplus \widetilde{\mathcal{H}}_{b}}_{\dot{\mathcal{H}}_{0}}
\end{aligned}
$$

In (3.22) and (3.23) the operators $M_{4 j}(\lambda), j \in\{1, \ldots, 4\}$, acting by definition (3.5) to $\mathcal{H}_{b}$ are considered as acting to $\widetilde{\mathcal{H}}_{b}$ (recall that $\mathcal{H}_{b} \subset \widetilde{\mathcal{H}}_{b}$ and $\left.\widetilde{\mathcal{H}}_{b}=\mathcal{H}_{b} \oplus \mathcal{H}_{b}^{\perp}\right)$. 
It follows from Proposition 4.9 and Lemma 4.14, $(2)$ in [35] that $T_{\theta^{\times}}$ is a symmetric extension of $T_{\min }$ and $\dot{\mathcal{M}}(\cdot)$ is the operator function $(2.10)$ of a certain boundary triplet $\dot{\Pi}=\left\{\dot{\mathcal{H}}_{0} \oplus \dot{\mathcal{H}}_{1}, \dot{\Gamma}_{0}, \dot{\Gamma}_{1}\right\}$ for $\left(T_{\theta^{\times}}\right)^{*}$. Therefore $\dot{\mathcal{M}}(\cdot) \in R_{u}\left[\dot{\mathcal{H}}_{0}\right]$.

Definition 3.10. An operator pair

$$
\widetilde{\tau}=\widetilde{\tau}(\lambda)=\left\{\widetilde{C}_{0}(\lambda), \widetilde{C}_{1}(\lambda)\right\} \in \widetilde{R}_{\dot{\mathcal{H}}_{1}}\left(\dot{\mathcal{H}}_{0}\right), \quad \lambda \in \mathbb{C}_{+},
$$

with holomorphic operator functions $\widetilde{C}_{j}(\cdot): \mathbb{C}_{+} \rightarrow\left[\dot{\mathcal{H}}_{0}\right], j \in\{0,1\}$, is called a boundary parameter.

It follows from (3.10) that in the case $N_{+}=N_{-}$(and only in this case) $\widetilde{\mathcal{H}}_{b}=\mathcal{H}_{b}, \dot{\mathcal{H}}_{0}=\dot{\mathcal{H}}_{1}=: \dot{\mathcal{H}}$ and $\widetilde{\tau} \in \widetilde{R}(\dot{\mathcal{H}})$.

Definition 3.11. A boundary parameter $\widetilde{\tau}$ of the form (3.24) is called admissible if

$$
\begin{aligned}
& \lim _{y \rightarrow+\infty} \frac{1}{i y} P_{\dot{\mathcal{H}}_{0}, \dot{\mathcal{H}}_{1}}\left(\widetilde{C}_{0}(i y)-\widetilde{C}_{1}(i y) \dot{\mathcal{M}}(i y)\right)^{-1} \widetilde{C}_{1}(i y) \uparrow \dot{\mathcal{H}}_{1}=0 \\
& \lim _{y \rightarrow+\infty} \frac{1}{i y} P_{\dot{\mathcal{H}}_{0}, \dot{\mathcal{H}}_{1}} \dot{\mathcal{M}}(i y)\left(\widetilde{C}_{0}(i y)-\widetilde{C}_{1}(i y) \dot{\mathcal{M}}(i y)\right)^{-1} \widetilde{C}_{0}(i y) \uparrow \dot{\mathcal{H}}_{1}=0
\end{aligned}
$$

In the following with the operator $\widetilde{U}$ from the assumption (A2) we associate the operator $U=U_{\theta} \in\left[\mathbb{H}_{0}, \mathbb{H}\right]$ given by

$$
U=U_{\theta}:=\widetilde{U} \uparrow \mathbb{H}_{0}
$$

Clearly ker $U=\{0\}$ and $U \mathbb{H}_{0}=\theta$.

A parametrization of all pseudospectral and spectral functions $\sigma(\cdot)$ (with respect to $U \in\left[\mathbb{H}_{0}, \mathbb{H}\right]$ ) in terms of a boundary parameter $\widetilde{\tau}$ is given by the following theorem.

Theorem 3.12. Let the assumptions (A1)-(A3) be satisfied, let $\dot{\mathcal{H}}_{0}$ and $\dot{\mathcal{H}}_{1}$ be finite-dimensional Hilbert spaces (3.18) and let $m_{0}(\cdot), S_{1}(\cdot), S_{2}(\cdot)$ and $\dot{\mathcal{M}}(\cdot)$ be the operator-functions $(3.20)-(3.23)$. Then:

(1) The set of pseudospectral functions $\sigma(\cdot)$ of the system (3.1) (with respect to $\left.U \in\left[\mathbb{H}_{0}, \mathbb{H}\right]\right)$ is not empty and the equalities

$$
\begin{gathered}
m_{\widetilde{\tau}}(\lambda)=m_{0}(\lambda)+S_{1}(\lambda)\left(\widetilde{C}_{0}(\lambda)-\widetilde{C}_{1}(\lambda) \dot{\mathcal{M}}(\lambda)\right)^{-1} \widetilde{C}_{1}(\lambda) S_{2}(\lambda), \quad \lambda \in \mathbb{C}_{+} \\
\sigma_{\widetilde{\tau}}(s)=\lim _{\delta \rightarrow+0} \lim _{y \rightarrow+0} \frac{1}{\pi} \int_{-\delta}^{s-\delta} \operatorname{Im} m_{\widetilde{\tau}}(x+i y) d x
\end{gathered}
$$

establish a bijective correspondence $\sigma(s)=\sigma_{\widetilde{\tau}}(s)$ between all admissible boundary parameters $\widetilde{\tau}$ defined by (3.24) and all pseudospectral functions $\sigma(\cdot)$ (with respect to $U$ ). 
(2) The following statements are equivalent:

(a) all boundary parameters $\widetilde{\tau}$ are admissible;

(b) statement (1) is valid for arbitrary boundary parameters;

(c) $\operatorname{mul} T_{\theta^{\times}}=\operatorname{mul} T_{\theta^{\times}}^{*}$

(d) $\lim _{y \rightarrow+\infty} \frac{1}{i y} \dot{\mathcal{M}}(i y)=0$ and $\lim _{y \rightarrow+\infty} y \cdot \operatorname{Im}(\dot{\mathcal{M}}(i y) h, h)=+\infty, \quad 0 \neq$ $h \in \dot{\mathcal{H}}_{0}$.

(3) The set of spectral functions of the system (3.1) (with respect to $\left.U \in\left[\mathbb{H}_{0}, \mathbb{H}\right]\right)$ is not empty if and only if mul $T_{\theta^{\times}}=\{0\}$. If this condition is satisfied, then the sets of spectral and pseudospectral functions of the system (3.1) coincide and hence statements (1) and (2) are valid for spectral functions (instead of pseudospectral ones).

Proof. (1) It follows from (3.5) and (3.22) that $S_{2}(\lambda) \mathbb{H}_{0} \subset \dot{\mathcal{H}}_{1} \subset \dot{\mathcal{H}}_{0}$. Therefore the equality

$$
\widetilde{S}_{2}(\lambda) h_{0}=S_{2}(\lambda) h_{0}\left(\in \dot{\mathcal{H}}_{1}\right), \quad h_{0} \in \mathbb{H}_{0}, \quad \lambda \in \mathbb{C}_{+}
$$

correctly defines the operator function $\widetilde{S}_{2}(\lambda) \in\left[\mathbb{H}_{0}, \dot{\mathcal{H}}_{1}\right]$. Moreover, let $\dot{M}_{+}(\lambda) \in\left[\dot{\mathcal{H}}_{0}, \dot{\mathcal{H}}_{1}\right]$ be given by the matrix in the right hand side of $(3.23)$ with $M_{44}$ in place of $M_{44}+\frac{i}{2} P_{\mathcal{H}_{b}^{\perp}}$. Then due to the decomposition (3.19) of $\dot{\mathcal{H}}_{0}$ one has

$$
\begin{gathered}
S_{2}(\lambda)=\left(\begin{array}{c}
\widetilde{S}_{2}(\lambda) \\
0
\end{array}\right): \mathbb{H}_{0} \rightarrow \dot{\mathcal{H}}_{1} \oplus \mathcal{H}_{b}^{\perp}, \\
\dot{\mathcal{M}}(\lambda)=\left(\begin{array}{c}
\dot{M}_{+}(\lambda) \\
\frac{i}{2} P_{\dot{\mathcal{H}}_{0}, \mathcal{H}_{b}^{\perp}}^{\perp}
\end{array}\right): \dot{\mathcal{H}}_{0} \rightarrow \dot{\mathcal{H}}_{1} \oplus \mathcal{H}_{b}^{\perp}
\end{gathered}
$$

and according to [35, Theorem 5.5] the equality

$$
m_{\widetilde{\tau}}(\lambda)=m_{0}(\lambda)+S_{1}(\lambda)\left(C_{0}(\lambda)-C_{1}(\lambda) \dot{M}_{+}(\lambda)\right)^{-1} C_{1}(\lambda) \widetilde{S}_{2}(\lambda), \quad \lambda \in \mathbb{C}_{+}
$$

together with (3.29) gives a bijective correspondence between all operator pairs

$$
\tau=\tau(\lambda)=\left\{C_{0}(\lambda), C_{1}(\lambda)\right\} \in \widetilde{R}\left(\dot{\mathcal{H}}_{0}, \dot{\mathcal{H}}_{1}\right)
$$

satisfying the conditions

$$
\begin{gathered}
\lim _{y \rightarrow+\infty} \frac{1}{i y} P_{\dot{\mathcal{H}}_{0}, \dot{\mathcal{H}}_{1}}\left(C_{0}(i y)-C_{1}(i y) \dot{M}_{+}(i y)\right)^{-1} C_{1}(i y)=0 \\
\lim _{y \rightarrow+\infty} \frac{1}{i y} \dot{M}_{+}(i y)\left(C_{0}(i y)-C_{1}(i y) \dot{M}_{+}(i y)\right)^{-1} C_{0}(i y)\left\lceil\dot{\mathcal{H}}_{1}=0\right.
\end{gathered}
$$


and all pseudospectral functions $\sigma(\cdot)$ of the system (3.1) (with respect to $\left.U \in\left[\mathbb{H}_{0}, \mathbb{H}\right]\right)$.

In view of Lemma 2.5, (2) the equalities (2.5), (2.6) and (2.7) (with $\dot{\mathcal{H}}_{j}$ instead of $\mathcal{H}_{j}$ ) give a bijective correspondence between all pairs $\tau$ of the form (3.33) and all boundary parameters $\widetilde{\tau}$ of the form (3.24). Moreover, using the second equality in (3.5) one gets

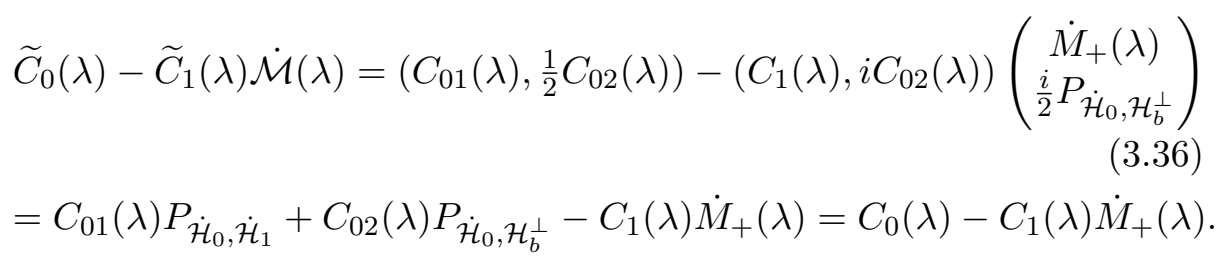

Therefore

$$
\begin{aligned}
& P_{\dot{\mathcal{H}}_{0}, \dot{\mathcal{H}}_{1}}\left(\widetilde{C}_{0}(\lambda)-\widetilde{C}_{1}(\lambda) \dot{\mathcal{M}}(\lambda)\right)^{-1} \widetilde{C}_{1}(\lambda) \uparrow \dot{\mathcal{H}}_{1} \\
& =P_{\dot{\mathcal{H}}_{0}, \dot{\mathcal{H}}_{1}}\left(C_{0}(\lambda)-C_{1}(\lambda) \dot{M}_{+}(\lambda)\right)^{-1} C_{1}(\lambda) ; \\
& P_{\dot{\mathcal{H}}_{0}, \dot{\mathcal{H}}_{1}} \dot{\mathcal{M}}(\lambda)\left(\widetilde{C}_{0}(\lambda)-\widetilde{C}_{1}(\lambda) \dot{\mathcal{M}}(\lambda)\right)^{-1} \widetilde{C}_{0}(\lambda)\left\lceil\dot{\mathcal{H}}_{1}\right. \\
& =\dot{M}_{+}(\lambda)\left(C_{0}(\lambda)-C_{1}(\lambda) \dot{M}_{+}(\lambda)\right)^{-1} C_{0}(\lambda) \uparrow \dot{\mathcal{H}}_{1}
\end{aligned}
$$

and hence the boundary parameter $\widetilde{\tau}$ is admissible if and only if the pair $\tau$ satisfies (3.34) and (3.35). Next, in view of (3.36) and the first equality in (3.5) one has

$$
\begin{gathered}
\left(\widetilde{C}_{0}(\lambda)-\widetilde{C}_{1}(\lambda) \dot{\mathcal{M}}(\lambda)\right)^{-1} \widetilde{C}_{1}(\lambda) S_{2}(\lambda) \\
=\left(C_{0}(\lambda)-C_{1}(\lambda) \dot{M}_{+}(\lambda)\right)^{-1}\left(C_{1}(\lambda), i C_{02}(\lambda)\right)\left(\begin{array}{c}
\widetilde{S}_{2}(\lambda) \\
0
\end{array}\right) \\
=\left(C_{0}(\lambda)-C_{1}(\lambda) \dot{M}_{+}(\lambda)\right)^{-1} C_{1}(\lambda) \widetilde{S}_{2}(\lambda) .
\end{gathered}
$$

Therefore equality (3.32) can be written in the form (3.28), which proves the first assertion of statement (1). The second assertion is implied by [35, Corollary 5.7].

Statement (2) follows from [35, Theorem 5.8] and representation (3.5) of $\dot{\mathcal{M}}(\lambda)$.

Statement (3) is implied by Proposition 3.4.

Remark 3.13. Note that $m_{\widetilde{\tau}}(\cdot)$ in $(3.28)$ is an $\left[\mathbb{H}_{0}\right]$-valued Nevanlinna function (the $m$-function of the system, see [35]) and (3.29) is the Stieltjes inversion formula for $m_{\widetilde{\tau}}(\cdot)$.

Lemma 3.14. The following statements are equivalent:

(1) The equality $\lim _{y \rightarrow+\infty} \frac{1}{i y} \dot{\mathcal{M}}(i y)=0$ holds.

(2) For each boundary parameter $\widetilde{\tau}$ of the form (3.24) its admissibility is equivalent to unique condition (3.25). 
Proof. As was shown in the proof of Theorem 3.12 a boundary parameter $\widetilde{\tau}$ is admissible if and only if the respective pair $\tau$ of the form (3.33) satisfies (3.34) and (3.35). Now the required statement follows from [35, Proposition 5.2] and [31, Theorems 4.6 and 4.9].

\section{Pseudospectral functions of symmetric systems in the case $N_{+}=n$}

\subsection{The monodromy matrix and the matrix $W(\lambda)$}

Within this section we consider symmetric systems (3.1) with the maximally possible deficiency index $N_{+}=n$.

Lemma 4.1. If $N_{+}=n$, then there exist a subspace $H^{\prime} \subset H$ and an operator

$$
\Gamma_{b}=\left(\Gamma_{0 b}, \widehat{\Gamma}_{b}, \Gamma_{1 b}\right)^{\top}: \operatorname{dom} \mathcal{T}_{\max } \rightarrow H \oplus \widehat{H} \oplus H
$$

such that

$$
\begin{gathered}
P_{H, H^{\prime}} \Gamma_{1 b}=-\frac{i}{2} P_{H, H^{\prime}} \Gamma_{0 b} \\
\operatorname{ran} \Gamma_{b}=\left\{h \oplus \widehat{h} \oplus h^{\prime} \in H \oplus \widehat{H} \oplus H: P_{H, H^{\prime}} h^{\prime}=-\frac{i}{2} P_{H, H^{\prime}} h\right\} \\
{[y, z]_{b}=\left(J \Gamma_{b} y, \Gamma_{b} z\right)=\left(\Gamma_{0 b} y, \Gamma_{1 b} z\right)-\left(\Gamma_{1 b} y, \Gamma_{0 b} z\right)+i\left(\widehat{\Gamma}_{b} y, \widehat{\Gamma}_{b} z\right)}
\end{gathered}
$$

(the identity (4.3) holds for all $\left.y, z \in \operatorname{dom} \mathcal{T}_{\max }\right)$. Moreover, for each such a subspace $H^{\prime}$

$$
\operatorname{dim} H^{\prime}=n-N_{-} .
$$

Proof. Since $N_{-} \leq n=N_{+}$, it follows from Lemma 3.8 that there exist finite dimensional Hilbert spaces $\widetilde{\mathcal{H}}_{b}$ and $\mathcal{H}_{b} \subset \widetilde{\mathcal{H}}_{b}$ and a surjective operator $\Gamma_{b}^{\prime}$ of the form (3.8) satisfying (3.9). Moreover, by the first equality in (3.10) $\operatorname{dim} \widetilde{\mathcal{H}}_{b}=\operatorname{dim} H$ and hence one can put $\widetilde{\mathcal{H}}_{b}=H$. Next assume that $H^{\prime}:=\mathcal{H}_{b}^{\perp}$. Then the immediate checking shows that the operator (4.1) with $\Gamma_{0 b}=\Gamma_{0 b}^{\prime}, \widehat{\Gamma}_{b}=\widehat{\Gamma}_{b}^{\prime}$ and $\Gamma_{1 b}=\Gamma_{1 b}^{\prime}-\frac{i}{2} P_{H^{\prime}} \Gamma_{0 b}^{\prime}$ satisfies (4.2)-(4.4). The last statement of the lemma directly follows from (3.10).

Assume that $N_{+}=n$. Let $H^{\prime}$ be a subspace in $H$ and let $\Gamma_{b}$ be operator (4.1) satisfying (4.2)-(4.4). Moreover, let $\widetilde{U} \in[\mathbb{H}]$ be an operator such that $\widetilde{U}^{*} J \widetilde{U}=J$ and let $Y_{\widetilde{U}}(\cdot, \lambda)$ be the $[\mathbb{H}]$-valued operator solution of $(3.1)$ with $Y_{\widetilde{U}}(a, \lambda)=\widetilde{U}, \lambda \in \mathbb{C}$. Since $N_{+}=n$, it follows that $Y_{\widetilde{U}}(\cdot, \lambda) \in \mathcal{L}_{\Delta}^{2}[\mathbb{H}], \lambda \in \mathbb{C}_{+}$. This fact enables us to introduce the following definition. 
Definition 4.2. The (holomorphic) operator function $B=B(\cdot): \mathbb{C}_{+} \rightarrow$ [H] defined for all $\lambda \in \mathbb{C}_{+}$by

$$
B(\lambda)=\Gamma_{b} Y_{\widetilde{U}}(\lambda)
$$

will be called the monodromy matrix of the system (3.1).

Below within this subsection we suppose the following assumptions:

(B1) For system (3.1) the equality $N_{+}=n$ is valid.

(B2) $\theta$ is a subspace in $\mathbb{H}$ such that $\theta^{\times} \in \operatorname{Sym}(\mathbb{H})$. Moreover, the assumption (A2) from Section 3.4 is fulfilled.

(B3) $H^{\prime}$ is a subspace in $H$ and $\Gamma_{b}$ is the operator (4.1) satisfying (4.2)-(4.4). Moreover, $B(\cdot)$ is the monodromy matrix (4.6).

Assumption (B2) implies that $B(\cdot)$ admits the block representation

$$
\begin{aligned}
& B(\lambda)=\left(\begin{array}{lllll}
B_{11} & B_{12} & B_{13} & B_{14} & B_{15} \\
B_{21} & B_{22} & B_{23} & B_{24} & B_{25} \\
B_{31} & B_{32} & B_{33} & B_{34} & B_{35}
\end{array}\right): \underbrace{H}_{\underbrace{\perp}_{H} \oplus H_{1} \oplus \widehat{H} \oplus \overbrace{H_{1} \oplus H_{1}^{\perp}}^{H}} \\
& \rightarrow \underbrace{H \oplus \widehat{H} \oplus H}_{\mathbb{H}}
\end{aligned}
$$

with entries $B_{i j}=B_{i j}(\lambda), \lambda \in \mathbb{C}_{+}$. Since by (4.1)

$$
\Gamma_{0 b} B(\lambda)=\left(B_{11}, B_{12}, \ldots, B_{15}\right) \text { and } \Gamma_{1 b} B(\lambda)=\left(B_{31}, B_{32}, \ldots, B_{35}\right),
$$

it follows from (4.2) that

$$
P_{H, H^{\prime}} B_{3 j}(\lambda)=-\frac{i}{2} P_{H, H^{\prime}} B_{1 j}(\lambda), \quad j \in\{1,2, \ldots, 5\}, \quad \lambda \in \mathbb{C}_{+} .
$$

For each $\lambda \in \mathbb{C}_{+}$we put

$$
W(\lambda)=\left(\begin{array}{ll}
w_{1}(\lambda) & w_{2}(\lambda) \\
w_{3}(\lambda) & w_{4}(\lambda)
\end{array}\right): \mathbb{H}_{0} \oplus \mathbb{H}_{0} \rightarrow \mathbb{H}_{0} \oplus \mathbb{H}_{0},
$$


where $w_{j}=w_{j}(\lambda)$ are defined in terms of $B_{i j}=B_{i j}(\lambda)$ by $w_{1}(\lambda)$

$$
\begin{gathered}
=\left(\begin{array}{cccc}
B_{11} & B_{12} & B_{13} & B_{14} \\
-i B_{21} & -i B_{22} & -i\left(B_{23}-I_{\widehat{H}}\right) & -i B_{24} \\
0 & 0 & 0 & -I_{H_{1}}
\end{array}\right): \underbrace{H_{1}^{\perp} \oplus H_{1} \oplus \widehat{H} \oplus H_{1}}_{\mathbb{H}_{0}} \\
\rightarrow \underbrace{H \oplus \widehat{H} \oplus H_{1}}_{\mathbb{H}_{0}}
\end{gathered}
$$

$w_{2}(\lambda)$

$$
\begin{gathered}
=\left(\begin{array}{cccc}
B_{15} & \frac{1}{2} B_{14} & \frac{i}{2} B_{13} & -\frac{1}{2} B_{12} \\
-i B_{25} & -\frac{i}{2} B_{24} & \frac{1}{2}\left(B_{23}+I_{\widehat{H}}\right) & \frac{i}{2} B_{22} \\
0 & \frac{1}{2} I_{H_{1}} & 0 & 0
\end{array}\right): H_{1}^{\perp} \oplus H_{1} \oplus \widehat{H} \oplus H_{1} \\
\rightarrow H \oplus \widehat{H} \oplus H_{1}
\end{gathered}
$$

$w_{3}(\lambda)$

$$
\begin{gathered}
=\left(\begin{array}{cccc}
B_{31} & B_{32} & B_{33} & B_{34} \\
-\frac{1}{2} B_{21} & -\frac{1}{2} B_{22} & -\frac{1}{2}\left(B_{23}+I_{\widehat{H}}\right) & -\frac{1}{2} B_{24} \\
0 & -I_{H_{1}} & 0 & 0
\end{array}\right): H_{1}^{\perp} \oplus H_{1} \oplus \widehat{H} \oplus H_{1} \\
\rightarrow H \oplus \widehat{H} \oplus H_{1}
\end{gathered}
$$

$w_{4}(\lambda)$

$$
\begin{gathered}
=\left(\begin{array}{cccc}
B_{35} & \frac{1}{2} B_{34} & \frac{i}{2} B_{33} & -\frac{1}{2} B_{32} \\
-\frac{1}{2} B_{25} & -\frac{1}{4} B_{24} & -\frac{i}{4}\left(B_{23}-I_{\widehat{H}}\right) & \frac{1}{4} B_{22} \\
0 & 0 & 0 & -\frac{1}{2} I_{H_{1}}
\end{array}\right): H_{1}^{\perp} \oplus H_{1} \oplus \widehat{H} \oplus H_{1} \\
\rightarrow H \oplus \widehat{H} \oplus H_{1}
\end{gathered}
$$

Clearly, $W(\cdot)$ is a holomorphic operator function defined on $\mathbb{C}_{+}$with values in $\left[\mathbb{H}_{0} \oplus \mathbb{H}_{0}\right]$.

Let in addition to the assumptions (B1)-(B3) system (3.1) be $\theta$ definite and let $\mathcal{H}_{b}=H \ominus H^{\prime}$ (so that $\mathcal{H}_{b}^{\perp}=H^{\prime}$ ) and

$$
\Gamma_{b}^{\prime}=\left(\Gamma_{0 b}, \widehat{\Gamma}_{b}, P_{H, \mathcal{H}_{b}} \Gamma_{1 b}\right)^{\top}: \operatorname{dom} \mathcal{T}_{\max } \rightarrow H \oplus \widehat{H} \oplus \mathcal{H}_{b} .
$$

Using (4.2)-(4.4) one can easily check that $\Gamma_{b}^{\prime}$ is a surjective operator satisfying (3.9) and hence assumptions (A1)-(A3) in section 3.4 are satisfied (with $\Gamma_{b}^{\prime}$ of the form (4.14)). Therefore by Proposition 3.9 the equalities (3.13)-(3.15) (with $\widetilde{\mathcal{H}}_{b}=H$ and $\mathcal{H}_{b}=H \ominus H^{\prime}$ ) define a decomposing 
boundary pair $\left\{\mathcal{H}_{0} \oplus \mathcal{H}_{1}, \Gamma\right\}$ for $T_{\max }$ and the Weyl function $M_{+}(\cdot)$ of this pair admits the block representation (3.5) with $\widetilde{\mathcal{H}}_{b}=H$. In this case the spaces $\dot{\mathcal{H}}_{0}$ and $\dot{\mathcal{H}}_{1}$ take the form

$$
\dot{\mathcal{H}}_{0}=H_{1} \oplus \widehat{H} \oplus H, \quad \dot{\mathcal{H}}_{1}=H_{1} \oplus \widehat{H} \oplus \mathcal{H}_{b}
$$

and the equalities (3.20)-(3.23) define the operator functions $m_{0}, S_{1}, S_{2}$ and $\dot{\mathcal{M}}$. Moreover, the decomposition (3.19) takes the form

$$
\dot{\mathcal{H}}_{0}=\dot{\mathcal{H}}_{1} \oplus H^{\prime} .
$$

Proposition 4.3. Assume that system (3.1) is $\theta$-definite. Moreover, let $U_{0} \in\left[\dot{\mathcal{H}}_{0}, \mathbb{H}_{0}\right]$ be a unitary operator given by

$$
U_{0}=\left(\begin{array}{ccc}
0 & 0 & I_{H} \\
0 & I_{\widehat{H}} & 0 \\
I_{H_{1}} & 0 & 0
\end{array}\right): \underbrace{H_{1} \oplus \widehat{H} \oplus H}_{\dot{\mathcal{H}}_{0}} \rightarrow \underbrace{H \oplus \widehat{H} \oplus H_{1}}_{\mathbb{H}_{0}} .
$$

Then for each $\lambda \in \mathbb{C}_{+}$the operator $S_{1}(\lambda)$ is invertible and the operator function $W=W(\lambda)$ admits the block representation

$$
\begin{gathered}
W=\left(\begin{array}{ll}
w_{1} & w_{2} \\
w_{3} & w_{4}
\end{array}\right) \\
=\left(\begin{array}{cc}
U_{0} S_{1}^{-1}(\lambda) & U_{0} S_{1}^{-1}(\lambda) m_{0}(\lambda) \\
-U_{0} \dot{\mathcal{M}}(\lambda) S_{1}^{-1}(\lambda) & U_{0}\left(S_{2}(\lambda)-\dot{\mathcal{M}}(\lambda) S_{1}^{-1}(\lambda) m_{0}(\lambda)\right)
\end{array}\right) .
\end{gathered}
$$

Proof. Assume that

$$
\begin{gathered}
\Omega_{0}(\lambda)=\left(\begin{array}{cc}
m_{0}(\lambda) & -\frac{1}{2} I_{H_{1}^{\perp}, \mathbb{H}_{0}} \\
-\frac{1}{2} P_{\mathbb{H}_{0}, H_{1}^{\perp}} & 0
\end{array}\right): \mathbb{H}_{0} \oplus H_{1}^{\perp} \rightarrow \mathbb{H}_{0} \oplus H_{1}^{\perp} \\
\widehat{S}_{1}(\lambda)=\left(\begin{array}{cc}
N_{1}(\lambda) & S_{1}(\lambda) \\
-I_{H_{1}^{\perp}} & 0
\end{array}\right): H_{1}^{\perp} \oplus \dot{\mathcal{H}}_{0} \rightarrow \mathbb{H}_{0} \oplus H_{1}^{\perp} \\
\widehat{S}_{2}(\lambda)=\left(\begin{array}{cc}
N_{2}(\lambda) & -I_{H_{1}^{\perp}} \\
\widetilde{S}_{2}(\lambda) & 0
\end{array}\right): \mathbb{H}_{0} \oplus H_{1}^{\perp} \rightarrow H_{1}^{\perp} \oplus \dot{\mathcal{H}}_{1},
\end{gathered}
$$

where $\widetilde{S}_{2}(\lambda)$ is taken from the block representation (3.5) of $S_{2}(\lambda)$ and

$$
\begin{gathered}
N_{1}(\lambda)=\left(M_{11}, M_{21}, M_{31}, 0\right)^{\top}: H_{1}^{\perp} \rightarrow H_{1}^{\perp} \oplus H_{1} \oplus \widehat{H} \oplus H_{1} \\
N_{2}(\lambda)=\left(M_{11}, M_{12}, M_{13}, 0\right): H_{1}^{\perp} \oplus H_{1} \oplus \widehat{H} \oplus H_{1} \rightarrow H_{1}^{\perp} .
\end{gathered}
$$

Moreover, let

$$
D=\operatorname{diag}\left(\frac{1}{2} I_{H_{1}^{\perp}}, I_{H_{1}}, I_{\widehat{H}}, I_{H_{1}}\right) \in\left[H_{1}^{\perp} \oplus H_{1} \oplus \widehat{H} \oplus H_{1}\right] .
$$


It follows from [33, Proposition 4.1] that the operator $\widehat{S}_{1}(\lambda)$ is invertible and

$$
\begin{gathered}
\widehat{S}_{1}^{-1}(\lambda)=\widetilde{w}_{1}(\lambda), \quad \widehat{S}_{1}^{-1}(\lambda) \Omega_{0}(\lambda)=\widetilde{w}_{2}(\lambda) \\
-M_{+}(\lambda) \widehat{S}_{1}^{-1}(\lambda)=\widetilde{w}_{3}(\lambda), \quad \widehat{S}_{2}(\lambda)-M_{+}(\lambda) \widehat{S}_{1}^{-1}(\lambda) \Omega_{0}(\lambda)=\widetilde{w}_{4}(\lambda),
\end{gathered}
$$

where

$$
\begin{aligned}
& \widetilde{w}_{1}(\lambda)=\left(\begin{array}{cc}
0 & -I_{H_{1}^{\perp}} \\
U_{0}^{-1} w_{1}(\lambda) & *
\end{array}\right): \mathbb{H}_{0} \oplus H_{1}^{\perp} \rightarrow H_{1}^{\perp} \oplus \dot{\mathcal{H}}_{0} \\
& \widetilde{w}_{2}(\lambda)=\left(\begin{array}{cc}
* & 0 \\
U_{0}^{-1} w_{2}(\lambda) D & *
\end{array}\right): \mathbb{H}_{0} \oplus H_{1}^{\perp} \rightarrow H_{1}^{\perp} \oplus \dot{\mathcal{H}}_{0} \\
& \widetilde{w}_{3}(\lambda)=\left(\begin{array}{cc}
* & 0 \\
P_{\dot{\mathcal{H}}_{0}, \dot{\mathcal{H}}_{1}} U_{0}^{-1} w_{3}(\lambda) & *
\end{array}\right): \mathbb{H}_{0} \oplus H_{1}^{\perp} \rightarrow H_{1}^{\perp} \oplus \dot{\mathcal{H}}_{1}
\end{aligned}
$$

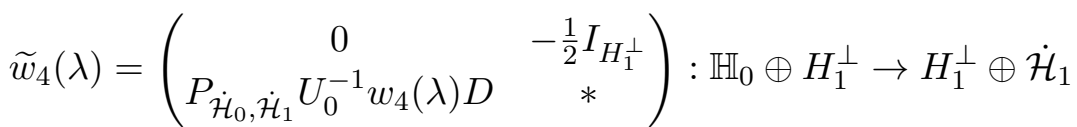

(the entries $*$ do not matter in further considerations). Since the operator $\widehat{S}_{1}(\lambda)$ is invertible, it follows from (4.20) that the operator $S_{1}(\lambda)$ is invertible and

$$
\widehat{S}_{1}^{-1}(\lambda)=\left(\begin{array}{cc}
0 & -I_{H_{1}^{\perp}} \\
S_{1}^{-1}(\lambda) & S_{1}^{-1}(\lambda) N_{1}(\lambda)
\end{array}\right): \mathbb{H}_{0} \oplus H_{1}^{\perp} \rightarrow H_{1}^{\perp} \oplus \dot{\mathcal{H}}_{0} .
$$

Combining this equality with the first equality in (4.24) and (4.26) one gets

$$
S_{1}^{-1}(\lambda)=U_{0}^{-1} w_{1}(\lambda)
$$

Clearly, the equality (3.5) can be written as

$$
M_{+}(\lambda)=\left(\begin{array}{cc}
* & * \\
\widehat{N}_{2}(\lambda) & \dot{M}_{+}(\lambda)
\end{array}\right): H_{1}^{\perp} \oplus \dot{\mathcal{H}}_{0} \rightarrow H_{1}^{\perp} \oplus \dot{\mathcal{H}}_{1},
$$

where $\dot{M}_{+}(\lambda)$ is taken from the block representation $(3.5)$ of $\dot{\mathcal{M}}(\lambda)$ and

$$
\widehat{N}_{2}(\lambda)=\left(M_{21}, M_{31}, M_{41}\right)^{\top}: H_{1}^{\perp} \rightarrow H_{1} \oplus \widehat{H} \oplus \mathcal{H}_{b} .
$$

Next we show that

$$
\begin{aligned}
& -\dot{M}_{+}(\lambda) S_{1}^{-1}(\lambda)=P_{\dot{\mathcal{H}}_{0}, \dot{\mathcal{H}}_{1}} U_{0}^{-1} w_{3}(\lambda), \\
& -\frac{i}{2} P_{\dot{\mathcal{H}}_{0}, H^{\prime}} S_{1}^{-1}(\lambda)=P_{\dot{\mathcal{H}}_{0}, H^{\prime}} U_{0}^{-1} w_{3}(\lambda) .
\end{aligned}
$$


It follows from (4.30) and (4.32) that

$$
-M_{+}(\lambda) \widehat{S}_{1}^{-1}(\lambda)=\left(\begin{array}{cc}
* & * \\
-\dot{M}_{+}(\lambda) S_{1}^{-1}(\lambda) & *
\end{array}\right) .
$$

Comparing this equality with the first equality in (4.25) and (4.28) one gets the first equality in (4.1). Moreover, by (4.31) and (4.8)

$$
\begin{gathered}
-\frac{i}{2} P_{\dot{\mathcal{H}}_{0}, H^{\prime}} S_{1}^{-1}(\lambda)=-\frac{i}{2} P_{\dot{\mathcal{H}}_{0}, H^{\prime}} U_{0}^{-1} w_{1}(\lambda)=-\frac{i}{2} P_{H, H^{\prime}}\left(B_{11}, B_{12}, B_{13}, B_{14}\right) \\
=P_{H, H^{\prime}}\left(B_{31}, B_{32}, B_{33}, B_{34}\right)=P_{\dot{\mathcal{H}}_{0}, H^{\prime}} U_{0}^{-1} w_{3}(\lambda)
\end{gathered}
$$

which proves the second equality in (4.1). Now the equalities (4.1) with taking (3.5) and (4.16) into account give

$$
-\dot{\mathcal{M}}(\lambda) S_{1}^{-1}(\lambda)=U_{0}^{-1} w_{3}(\lambda) .
$$

Next we show that

$$
m_{0}(\lambda)-\frac{1}{2} N_{1}(\lambda) P_{\mathbb{H}_{0}, H_{1}^{\perp}}=m_{0}(\lambda) D, \quad \widetilde{S}_{2}(\lambda)-\frac{1}{2} \widehat{N}_{2}(\lambda) P_{\mathbb{H}_{0}, H_{1}^{\perp}}=\widetilde{S}_{2}(\lambda) D .
$$

Indeed, by (3.20) and (4.22)

$$
\begin{gathered}
m_{0}(\lambda)-\frac{1}{2} N_{1}(\lambda) P_{\mathbb{H}_{0}, H_{1}^{\perp}}=\left(\begin{array}{cccc}
M_{11} & * & * & * \\
M_{21} & * & * & * \\
M_{31} & * & * & * \\
0 & * & * & *
\end{array}\right)-\frac{1}{2}\left(\begin{array}{c}
M_{11} \\
M_{21} \\
M_{31} \\
0
\end{array}\right)\left(I_{H_{1}^{\perp}}, 0,0,0\right) \\
=\left(\begin{array}{cccc}
\frac{1}{2} M_{11} & * & * & * \\
\frac{1}{2} M_{21} & * & * & * \\
\frac{1}{2} M_{31} & * & * & * \\
0 & * & * & *
\end{array}\right)=m_{0}(\lambda) D
\end{gathered}
$$

Moreover, by definition (3.30) the block representation of $\widetilde{S}_{2}(\lambda)$ is given by the right hand side of $(3.22)$ (with $\mathcal{H}_{b}$ instead of $\widetilde{\mathcal{H}}_{b}$ ). This and (4.33) yield

$$
\begin{gathered}
\widetilde{S}_{2}(\lambda)-\frac{1}{2} \widehat{N}_{2}(\lambda) P_{\mathbb{H}_{0}, H_{1}^{\perp}}=\left(\begin{array}{llll}
M_{21} & * & * & * \\
M_{31} & * & * & * \\
M_{41} & * & * & *
\end{array}\right)-\frac{1}{2}\left(\begin{array}{c}
M_{21} \\
M_{31} \\
M_{41}
\end{array}\right)\left(I_{H_{1}^{\perp}}, 0,0,0\right) \\
=\left(\begin{array}{cccc}
\frac{1}{2} M_{21} & * & * & * \\
\frac{1}{2} M_{31} & * & * & * \\
\frac{1}{2} M_{41} & * & * & *
\end{array}\right)=\widetilde{S}_{2}(\lambda) D .
\end{gathered}
$$


It follows from (4.30), (4.19) and the first equality in (4.36) that

$$
\begin{aligned}
\widehat{S}_{1}^{-1}(\lambda) \Omega_{0}(\lambda) & =\left(\begin{array}{cc}
\frac{1}{2} P_{\mathbb{H}_{0}, H_{1}^{\perp}} & * \\
S_{1}^{-1}(\lambda)\left(m_{0}(\lambda)-\frac{1}{2} N_{1}(\lambda) P_{\mathbb{H}_{0}, H_{1}^{\perp}}\right) & *
\end{array}\right) \\
& =\left(\begin{array}{cc}
\frac{1}{2} P_{\mathbb{H}_{0}, H_{1}^{\perp}} & * \\
S_{1}^{-1}(\lambda) m_{0}(\lambda) D & *
\end{array}\right) .
\end{aligned}
$$

Comparing this equality with the second equality in (4.24) and (4.27) one gets

$$
S_{1}^{-1}(\lambda) m_{0}(\lambda)=U_{0}^{-1} w_{2}(\lambda)
$$

Next we prove the equalities

$$
\begin{aligned}
& \widetilde{S}_{2}(\lambda)-\dot{M}_{+}(\lambda) S_{1}^{-1}(\lambda) m_{0}(\lambda)=P_{\dot{\mathcal{H}}_{0}, \dot{\mathcal{H}}_{1}} U_{0}^{-1} w_{4}(\lambda) \\
& -\frac{i}{2} P_{\dot{\mathcal{H}}_{0}, H^{\prime}} S_{1}^{-1}(\lambda) m_{0}(\lambda)=P_{\dot{\mathcal{H}}_{0}, H^{\prime}} U_{0}^{-1} w_{4}(\lambda) .
\end{aligned}
$$

It follows from (4.21), (4.32) and (4.1) that

$$
\begin{gathered}
\widehat{S}_{2}(\lambda)-M_{+}(\lambda) \widehat{S}_{1}^{-1}(\lambda) \Omega_{0}(\lambda) \\
=\left(\begin{array}{c}
* \\
\widetilde{S}_{2}(\lambda)-\frac{1}{2} \widehat{N}_{2}(\lambda) P_{\mathbb{H}_{0}, H_{1}^{\perp}}-\dot{M}_{+}(\lambda) S_{1}^{-1}(\lambda) m_{0}(\lambda) D
\end{array}\right) .
\end{gathered}
$$

Combining this equality with the second equality in (4.25), (4.29) and taking the second equality in (4.36) into account we obtain (4.39). Moreover, by (4.38) and (4.8)

$$
\begin{gathered}
-\frac{i}{2} P_{\dot{\mathcal{H}}_{0}, H^{\prime}} S_{1}^{-1}(\lambda) m_{0}(\lambda)=-\frac{i}{2} P_{\dot{\mathcal{H}}_{0}, H^{\prime}} U_{0}^{-1} w_{2}(\lambda) \\
=-\frac{i}{2} P_{H, H^{\prime}}\left(B_{15}, \frac{1}{2} B_{14}, \frac{i}{2} B_{13},-\frac{1}{2} B_{12}\right) \\
=P_{H, H^{\prime}}\left(B_{35}, \frac{1}{2} B_{34}, \frac{i}{2} B_{33},-\frac{1}{2} B_{32}\right)=P_{\dot{\mathcal{H}}_{0}, H^{\prime}} U_{0}^{-1} w_{4}(\lambda),
\end{gathered}
$$

which proves (4.40). Now the equalities (4.39) and (4.40) with taking the block representations $(3.5)$ of $S_{2}(\lambda)$ and $\dot{\mathcal{M}}(\lambda)$ into account give

$$
S_{2}(\lambda)-\dot{\mathcal{M}}(\lambda) S_{1}^{-1}(\lambda) m_{0}(\lambda)=U_{0}^{-1} w_{4}(\lambda) .
$$

Finally, combining (4.9) with (4.31), (4.35), (4.38) and (4.41) we arrive at (4.3).

Proposition 4.4. Assume that system (3.1) is $\theta$-definite. Moreover, let $Y_{U}(\cdot, \lambda) \in \mathcal{L}_{\Delta}^{2}\left[\mathbb{H}_{0}, \mathbb{H}\right]$ and $\psi(\cdot, \lambda) \in \mathcal{L}_{\Delta}^{2}\left[\mathbb{H}_{0}, \mathbb{H}\right], \lambda \in \mathbb{C}$, be the operator 
solutions of the system satisfying $Y_{U}(a, \lambda)=U\left(\Leftrightarrow \widetilde{U}^{-1} Y_{U}(a, \lambda)=I_{\mathbb{H}_{0}, \mathbb{H}}\right)$ and

$$
\begin{array}{r}
\widetilde{U}^{-1} \psi(a, \lambda)=\left(\begin{array}{cccc}
0 & 0 & 0 & 0 \\
0 & 0 & 0 & -\frac{1}{2} I_{H_{1}} \\
0 & 0 & \frac{i}{2} I_{\widehat{H}} & 0 \\
0 & \frac{1}{2} I_{H_{1}} & 0 & 0 \\
I_{H_{1}^{\perp}} & 0 & 0 & 0
\end{array}\right): \underbrace{H_{1}^{\perp} \oplus H_{1} \oplus \widehat{H} \oplus H_{1}}_{\mathbb{H}_{0}} \\
\rightarrow \underbrace{H_{1}^{\perp} \oplus H_{1} \oplus \widehat{H} \oplus H_{1} \oplus H_{1}^{\perp}}_{\mathbb{H}} \cdot
\end{array}
$$

Then for all $\lambda, \mu \in \mathbb{C}_{+}$the following identities hold:

$$
\begin{gathered}
-w_{1}^{*}(\lambda) w_{3}(\mu)+w_{3}^{*}(\lambda) w_{1}(\mu)=(\mu-\bar{\lambda}) \int_{\mathcal{I}} Y_{U}^{*}(t, \lambda) \Delta(t) Y_{U}(t, \mu) d t, \\
-w_{2}^{*}(\lambda) w_{3}(\mu)+w_{4}^{*}(\lambda) w_{1}(\mu)-I_{\mathbb{H}_{0}}=(\mu-\bar{\lambda}) \int_{\mathcal{I}} \psi^{*}(t, \lambda) \Delta(t) Y_{U}(t, \mu) d t, \\
-w_{2}^{*}(\lambda) w_{4}(\mu)+w_{4}^{*}(\lambda) w_{2}(\mu)=(\mu-\bar{\lambda}) \int_{\mathcal{I}} \psi^{*}(t, \lambda) \Delta(t) \psi(t, \mu) d t .
\end{gathered}
$$

These identities mean that for all $\lambda, \mu \in \mathbb{C}_{+}$

$$
W^{*}(\lambda) J_{0} W(\mu)-J_{0}=i(\mu-\bar{\lambda}) \int_{\mathcal{I}} \eta^{*}(t, \lambda) \Delta(t) \eta(t, \mu) d t,
$$

where

$J_{0}=\left(\begin{array}{cc}0 & -i I_{\mathbb{H}_{0}} \\ i I_{\mathbb{H}_{0}} & 0\end{array}\right) \quad$ and $\quad \eta(t, \lambda)=\left(Y_{U}(t, \lambda), \psi(t, \lambda)\right): \mathbb{H}_{0} \oplus \mathbb{H}_{0} \rightarrow \mathbb{H}$.

Proof. Assume that

$$
\begin{aligned}
\left(\begin{array}{cc}
a_{1}(\lambda) & a_{2}(\lambda) \\
a_{3}(\lambda) & a_{4}(\lambda)
\end{array}\right) & :=\left(\begin{array}{cc}
m_{0}(\lambda) & S_{1}(\lambda) U_{0}^{-1} \\
U_{0} S_{2}(\lambda) & U_{0} \dot{\mathcal{M}}(\lambda) U_{0}^{-1}
\end{array}\right): \mathbb{H}_{0} \oplus \mathbb{H}_{0} \\
& \rightarrow \mathbb{H}_{0} \oplus \mathbb{H}_{0}, \quad \lambda \in \mathbb{C}_{+} .
\end{aligned}
$$

Moreover, let $\gamma_{+}(\cdot)$ be the $\gamma$-field of the decomposing boundary pair $\left\{\mathcal{H}_{0} \oplus \mathcal{H}_{1}, \Gamma\right\}$ for $T_{\max }$ (see Proposition 3.9), let

$$
\gamma_{+}(\lambda)=\left(\gamma_{1}(\lambda), \gamma_{2}(\lambda), \gamma_{3}(\lambda), \gamma_{4}(\lambda)\right): H_{1}^{\perp} \oplus H_{1} \oplus \widehat{H} \oplus H \rightarrow \mathfrak{H}, \quad \lambda \in \mathbb{C}_{+}
$$


be the block representation of $\gamma_{+}(\lambda)$ and let $\gamma_{0}(\lambda)\left(\in\left[\mathbb{H}_{0}, \mathfrak{H}\right]\right)$ and $\dot{\gamma}(\lambda)(\in$ $\left.\left[\mathbb{H}_{0}, \mathfrak{H}\right]\right)$ be the operator functions given by

$$
\begin{aligned}
\gamma_{0}(\lambda)= & \left(\gamma_{1}(\lambda), \gamma_{2}(\lambda), \gamma_{3}(\lambda), 0\right): H_{1}^{\perp} \oplus H_{1} \oplus \widehat{H} \oplus H_{1} \rightarrow \mathfrak{H} \\
\dot{\gamma}(\lambda)= & \left(\gamma_{4}(\lambda), \gamma_{3}(\lambda), \gamma_{2}(\lambda)\right): H \oplus \widehat{H} \oplus H_{1} \rightarrow \mathfrak{H} .
\end{aligned}
$$

By using identity (2.11) for the function $\mathcal{M}(\cdot)$ of the pair $\left\{\mathcal{H}_{0} \oplus \mathcal{H}_{1}, \Gamma\right\}$ one can easily prove that

$$
\begin{gathered}
a_{1}(\mu)-a_{1}^{*}(\lambda)=(\mu-\bar{\lambda}) \gamma_{0}^{*}(\lambda) \gamma_{0}(\mu), \quad a_{2}(\mu)-a_{3}^{*}(\lambda)=(\mu-\bar{\lambda}) \gamma_{0}^{*}(\lambda) \dot{\gamma}(\mu) \\
a_{4}(\mu)-a_{4}^{*}(\lambda)=(\mu-\bar{\lambda}) \dot{\gamma}^{*}(\lambda) \dot{\gamma}(\mu), \quad \mu, \lambda \in \mathbb{C}_{+} .
\end{gathered}
$$

Moreover, according to Proposition 4.3 equality (4.3) is valid. Therefore by [33, Lemma 4.2]

$$
\begin{gathered}
-w_{1}^{*}(\lambda) w_{3}(\mu)+w_{3}^{*}(\lambda) w_{1}(\mu)=(\mu-\bar{\lambda}) Q_{0}^{*}(\lambda) Q_{0}(\mu) \\
-w_{2}^{*}(\lambda) w_{3}(\mu)+w_{4}^{*}(\lambda) w_{1}(\mu)-I_{\mathbb{H}_{0}}=(\mu-\bar{\lambda}) Q_{1}^{*}(\lambda) Q_{0}(\mu), \\
-w_{2}^{*}(\lambda) w_{4}(\mu)+w_{4}^{*}(\lambda) w_{2}(\mu)=(\mu-\bar{\lambda}) Q_{1}^{*}(\lambda) Q_{1},(\mu), \quad \mu, \lambda \in \mathbb{C}_{+},
\end{gathered}
$$

where

$$
Q_{0}(\lambda)=\dot{\gamma}(\lambda) U_{0} S_{1}^{-1}(\lambda), \quad Q_{1}(\lambda)=-\gamma_{0}(\lambda)+Q_{0}(\lambda) m_{0}(\lambda), \quad \lambda \in \mathbb{C}_{+} .
$$

Next we show that

$$
Q_{0}(\lambda)=\pi_{\Delta} Y_{U}(\lambda), \quad Q_{1}(\lambda)=\pi_{\Delta} \psi(\lambda),
$$

where $Y_{U}(\lambda)$ and $\psi(\lambda)$ are operators (3.2) for solutions $Y_{U}(\cdot, \lambda)$ and $\psi(\cdot, \lambda)$. Similarly to $[33,(4.37)]$ one proves the equality

$$
\gamma_{+}(\lambda)=\pi_{\Delta} Y_{\widetilde{U}}(\lambda) \widehat{S}_{1}(\lambda), \quad \lambda \in \mathbb{C}_{+},
$$

where $\widehat{S}_{1}(\lambda)$ is given by (4.20) or, equivalently, by

$$
\begin{aligned}
\widehat{S}_{1}(\lambda)= & \left(\begin{array}{cccc}
M_{11} & M_{12} & M_{13} & M_{14} \\
M_{21} & M_{22} & M_{23} & M_{24} \\
M_{31} & M_{32} & M_{33}-\frac{i}{2} I_{\widehat{H}} & M_{34} \\
0 & -I_{H_{1}} & 0 & 0 \\
-I_{H_{1}^{\perp}} & 0 & 0 & 0
\end{array}\right): \underbrace{H_{1}^{\perp} \oplus H_{1} \oplus \widehat{H} \oplus H}_{\mathbb{H}} \\
& \rightarrow \underbrace{H_{1}^{\perp} \oplus H_{1} \oplus \widehat{H} \oplus H_{1} \oplus H_{1}^{\perp}}_{\mathbb{H}} .
\end{aligned}
$$


Moreover, by (4.48) and (4.49) $\dot{\gamma}(\lambda)=\left(\gamma_{+}(\lambda)\left\lceil\dot{\mathcal{H}}_{0}\right) U_{0}^{-1}, \quad \gamma_{0}(\lambda)=\right.$ $\gamma_{+}(\lambda) X$, where

$$
X=\operatorname{diag}\left(I_{H_{1}^{\perp}}, I_{H_{1}}, I_{\widehat{H}}, 0\right) \in\left[H_{1}^{\perp} \oplus H_{1} \oplus \widehat{H} \oplus H_{1}, H_{1}^{\perp} \oplus H_{1} \oplus \widehat{H} \oplus H\right] .
$$

Therefore in view of $(4.55)$

$$
\dot{\gamma}(\lambda)=\pi_{\Delta} Y_{\widetilde{U}}(\lambda)\left(\widehat{S}_{1}(\lambda)\left\lceil\dot{\mathcal{H}}_{0}\right) U_{0}^{-1}, \gamma_{0}(\lambda)=\pi_{\Delta} Y_{\widetilde{U}}(\lambda) \widehat{S}_{1}(\lambda) X, \quad \lambda \in \mathbb{C}_{+} .\right.
$$

It follows from (4.20) that $\widehat{S}_{1}(\lambda) \uparrow \dot{\mathcal{H}}_{0}=\left(S_{1}(\lambda), 0\right)^{\top}: \dot{\mathcal{H}}_{0} \rightarrow \mathbb{H}_{0} \oplus H_{1}^{\perp}$. Therefore by the first equalities in (4.53) and (4.56)

$$
\begin{gathered}
Q_{0}(\lambda)=\pi_{\Delta} Y_{\widetilde{U}}(\lambda)\left(\begin{array}{c}
S_{1}(\lambda) \\
0
\end{array}\right) S_{1}^{-1}(\lambda) \\
=\pi_{\Delta} Y_{\widetilde{U}}(\lambda) I_{\mathbb{H}_{0}, \mathbb{H}}=\pi_{\Delta} Y_{\widetilde{U}}(\lambda)\left(\widetilde{U}^{-1} Y_{U}(a, \lambda)\right) .
\end{gathered}
$$

Moreover, combining of the second equality in (4.53) with (4.1) and the second equality in (4.56) yields

$$
Q_{1}(\lambda)=\pi_{\Delta} Y_{\widetilde{U}}(\lambda)\left(-\widehat{S}_{1}(\lambda) X+I_{\mathbb{H}_{0}, \mathbb{H}} m_{0}(\lambda)\right)
$$

and the immediate calculation gives $-\widehat{S}_{1}(\lambda) X+I_{\mathbb{H}_{0}, \mathbb{H}} m_{0}(\lambda)=\widetilde{U}^{-1} \psi(a, \lambda)$, where $U^{-1} \psi(a, \lambda)$ is given by (4.42). Hence

$$
Q_{1}(\lambda)=\pi_{\Delta} Y_{\widetilde{U}}(\lambda)\left(\widetilde{U}^{-1} \psi(a, \lambda)\right)
$$

Since obviously

$$
Y_{\widetilde{U}}(t, \lambda)\left(\widetilde{U}^{-1} Y_{U}(a, \lambda)\right)=Y_{U}(t, \lambda) \text { and } Y_{\widetilde{U}}(t, \lambda)\left(\widetilde{U}^{-1} \psi(a, \lambda)\right)=\psi(t, \lambda),
$$

it follows that $Y_{\widetilde{U}}(\lambda)\left(\widetilde{U}^{-1} Y_{U}(a, \lambda)\right)=Y_{U}(\lambda)$ and $Y_{\widetilde{U}}(\lambda)\left(\widetilde{U}^{-1} \psi(a, \lambda)\right)=$ $\psi(\lambda)$. This and (4.1), (4.58) yield (4.54).

Next, application of [2, Lemma 3.3] to operators (4.54) gives

$$
\begin{gathered}
Q_{0}^{*}(\lambda) \widetilde{f}=\int_{\mathcal{I}} Y_{U}^{*}(t, \lambda) \Delta(t) f(t) d t \quad Q_{1}^{*}(\lambda) \widetilde{f}=\int_{\mathcal{I}} \psi^{*}(t, \lambda) \Delta(t) f(t) d t, \\
\tilde{f} \in \mathfrak{H}, \quad f(\cdot) \in \widetilde{f} .
\end{gathered}
$$

Therefore the equalities (4.50)-(4.52) can be written as (4.43)-(4.45).

The following corollary is immediate from Proposition 4.4.

Corollary 4.5. The operator function $W(\cdot)$ satisfies the inequality

$$
W^{*}(\lambda) J_{0} W(\lambda) \leq J_{0}, \quad \lambda \in \mathbb{C}_{+} .
$$


Remark 4.6. Let $\mathcal{H}$ be a finite dimensional Hilbert space and let $J \in[\mathcal{H}]$ be a signature operator (the latter means that $J^{*}=J^{-1}=J$ ). As is known (see e.g. [4]) the holomorphic operator (matrix) function $U(\cdot)$ : $\mathbb{C}_{+} \rightarrow[\mathcal{H}]$ is said to belong to the Potapov class $\mathcal{P}(J)$ if $U^{*}(\lambda) J U(\lambda) \leq$ $J, \lambda \in \mathbb{C}_{+}$. It follows from Corollary 4.5 that $W(\cdot) \in \mathcal{P}\left(J_{0}\right)$.

\subsection{Quasiregular and regular systems}

The following proposition is well known (see e.g. [27]).

Proposition 4.7. For system (3.1) the following assertions are equivalent:

(1) The system has maximal formal deficiency indices $N_{+}=N_{-}=n$.

(2) $\operatorname{dim} \mathcal{N}_{\lambda}=n$ for any $\lambda \in \mathbb{C}$.

(3) There exists $\lambda_{0} \in \mathbb{C}$ such that $\operatorname{dim} \mathcal{N}_{\lambda_{0}}=\operatorname{dim} \mathcal{N}_{\bar{\lambda}_{0}}=n$.

Definition 4.8. System (3.1) is said to be quasiregular if at least one (and hence all) of the conditions (1)-(3) are satisfied.

Definition 4.9. System (3.1) is called regular if $\mathcal{I}=[a, b]$ is a compact interval (and hence the coefficients $A(\cdot)$ and $\Delta(\cdot)$ are integrable on $\mathcal{I}$ ).

Remark 4.10. (1) Clearly, each regular system is quasiregular.

(2) Let system (3.1) be quasiregular and let $\Gamma_{b}$ be the operator (4.1) satisfying (4.2)-(4.4). Since by (4.5) $H^{\prime}=\{0\}$, it follows that $\Gamma_{b}$ is just a surjective operator satisfying (4.4). Moreover, in this case the monodromy matrix (4.6) is an entire operator function $B(\cdot): \mathbb{C} \rightarrow[\mathbb{H}]$.

Proposition 4.11. Assume that:

(BQ1) System (3.1) is quasiregular.

(BQ2) The assumption (B2) from Section 4.1 is satisfied.

(BQ3) $\Gamma_{b}$ is a surjective operator (4.1) satisfying (4.4) and $B(\cdot)$ is the monodromy matrix (4.6) with the block representation (4.1).

Then: (1) The equalities (4.9)-(4.13) define an entire function $W(\cdot)$ : $\mathbb{C} \rightarrow\left[\mathbb{H}_{0} \oplus \mathbb{H}_{0}\right]$.

(2) If in addition the system is $\theta$-definite, then the identities (4.43)(4.45) and (4.46) (with $J_{0}$ and $\eta(\cdot, \lambda)$ of the form (4.47)) hold for all $\lambda, \mu \in \mathbb{C}$. Moreover,

$$
W^{*}(\lambda) J_{0} W(\lambda) \leq J_{0}, \quad \lambda \in \mathbb{C}_{+} ; \quad W^{*}(\lambda) J_{0} W(\lambda)=J_{0}, \quad \lambda \in \mathbb{R},
$$

which implies that $W(\cdot)$ belongs to the class $\mathcal{U}\left(J_{0}\right)$ of $J_{0}$-inner operator (matrix) functions (for definition of this class see e.g. [4]). 
Proof. Statement (1) is obvious, wile statement (2) directly follows from Proposition 4.4.

For the regular system (3.1) one can put $\Gamma_{b} y=y(b), y \in \operatorname{dom} \mathcal{T}_{\max }$, in which case $B(\lambda)=Y_{\widetilde{U}}(b, \lambda)$ is the "classical" monodromy matrix (see e.g. [5]). An explicit construction of the operator $\Gamma_{b}$ and the monodromy matrix $B(\cdot)$ for the quasiregular system is suggested in our paper [33]. Namely, let system (3.1) be quasiregular and let an operator $\widetilde{U} \in[\mathbb{H}]$ satisfies $\widetilde{U}^{*} J \widetilde{U}=J$. Then according to [33] the equality

$$
\Gamma_{b} y:=\lim _{t \uparrow b} Y_{\widetilde{U}}^{-1}(t, 0) y(t)=\lim _{t \uparrow b}\left(-J Y_{\widetilde{U}}^{*}(t, 0) J y(t)\right), \quad y \in \operatorname{dom} \mathcal{T}_{\max },
$$

correctly defines a surjective operator $\Gamma_{b}: \operatorname{dom} \mathcal{T}_{\max } \rightarrow \mathbb{H}$ satisfying (4.4) and the respective monodromy matrix $B(\cdot)$ is

$$
B(\lambda)=\lim _{t \uparrow b}\left(-J Y_{\widetilde{U}}^{*}(t, 0) J Y_{\widetilde{U}}(t, \lambda)\right), \quad \lambda \in \mathbb{C} .
$$

An explicit representation of the operator function $W(\cdot)$ corresponding to $B(\lambda)$ of the form (4.61) is given in the following proposition.

Proposition 4.12. Let under the assumptions (BQ1) and (BQ2) of Proposition 4.11 the system be $\theta$-definite, let $B=B(\lambda)$ be the monodromy matrix (4.61) with the block representation (4.1) and let $W(\cdot)$ be the respective operator function (4.9)-(4.13). Assume also that $C=$ $\left(C_{i j}\right)_{i, j=1}^{2} \in\left[\mathbb{H}_{0} \oplus \mathbb{H}_{0}\right]$ is the operator matrix with the entries $C_{i j} \in\left[\mathbb{H}_{0}\right]$ given by the block representations

$$
\begin{array}{rlr}
C_{11}=\left(\begin{array}{ccc}
I_{H} & 0 & 0 \\
0 & 0 & 0 \\
0 & 0 & -I_{H_{1}}
\end{array}\right), & C_{12}=\left(\begin{array}{ccc}
0 & 0 & -\frac{1}{2} I_{H_{1}, H} \\
0 & I_{\widehat{H}} & 0 \\
\frac{1}{2} P_{H, H_{1}} & 0 & 0
\end{array}\right) \\
C_{21}=\left(\begin{array}{ccc}
0 & 0 & I_{H_{1}, H} \\
0 & -I_{\widehat{H}} & 0 \\
-P_{H, H_{1}} & 0 & 0
\end{array}\right), C_{22}=\left(\begin{array}{ccc}
P_{H_{1}^{\perp}}+\frac{1}{2} P_{H_{1}} & 0 & 0 \\
0 & 0 & 0 \\
0 & 0 & -\frac{1}{2} I_{H_{1}}
\end{array}\right),
\end{array}
$$

(with respect to the decomposition $\mathbb{H}_{0}=H \oplus \widehat{H} \oplus H_{1}$ ), $\eta(t, \lambda)$ is the operator solution (4.47) of (3.1) and $\widehat{\eta}(t, \lambda)=-i \eta(t, \lambda) J_{0} C^{*}$. Then $W(\cdot)$ admits the representation

$$
W(\lambda)=C+\lambda \int_{\mathcal{I}} \widehat{\eta}^{*}(t, 0) \Delta(t) \eta(t, \lambda) d t, \quad \lambda \in \mathbb{C} .
$$


Proof. Since by (4.46) $W^{*}(0) J_{0} W(0)=J_{0}$, it follows that $\left(W^{*}(0) J_{0}\right)^{-1}=$ $W(0) J_{0}$. This and (4.46) yield

$$
W(\lambda)=W(0)+i \lambda W(0) J_{0} \int_{\mathcal{I}} \eta^{*}(t, 0) \Delta(t) \eta(t, \lambda) d t, \quad \lambda \in \mathbb{C} .
$$

By (4.61) $B(0)=I_{\mathbb{H}}$ and therefore in (4.1) $B_{11}=B_{35}=I_{H_{1}^{\perp}, H}, B_{12}=$ $B_{34}=I_{H_{1}, H}, B_{23}=I_{\widehat{H}}$, while all other entries $B_{i j}$ are 0 . Hence by (4.10)-(4.13) $W(0)=C$ and the equality (4.63) yields (4.62).

Corollary 4.13. If in addition to the assumptions of Proposition 4.12 system is Hamiltonian and $\mathbb{H}_{0}=H \oplus\{0\}$, then equality (4.62) takes the form

$$
\begin{gathered}
W(\lambda)(=B(\lambda))=\left(\begin{array}{cc}
I_{H} & 0 \\
0 & I_{H}
\end{array}\right) \\
+\lambda \int_{\mathcal{I}}\left(\begin{array}{c}
\psi^{*}(t, 0) \\
-Y_{U}^{*}(t, 0)
\end{array}\right) \Delta(t)\left(Y_{U}(t, \mu), \psi(t, \mu)\right) d t
\end{gathered}
$$

where $Y_{U}(\cdot, \lambda)$ and $\psi(\cdot, \lambda)$ are the $[H, H \oplus H]$-valued solutions of $(3.1)$ given by

$$
\begin{gathered}
\widetilde{U}^{-1} Y_{U}(a, \lambda)=\left(I_{H}, 0\right)^{\top}: H \rightarrow H \oplus H, \\
\widetilde{U}^{-1} \psi(a, \lambda)=\left(0, I_{H}\right)^{\top}: H \rightarrow H \oplus H .
\end{gathered}
$$

If system (3.1) is quasiregular and the assumptions before (3.7) are fulfilled, then for each $\widetilde{f} \in \mathfrak{H}$ the equality (3.7) defines a continuous function $\widehat{f}(\cdot)=\widehat{f}_{\widehat{f}}(\cdot): \mathbb{R} \rightarrow \mathbb{H}_{0}^{\prime}$.

Proposition 4.14. Let system be quasiregular and let $\theta$ be a subspace in $\mathbb{H}$. Assume also that:

(i) $T_{\theta \times} \in \widetilde{\mathcal{C}}(\mathfrak{H})$ is linear relation (3.3) and mul $T_{\theta \times}$ is the multivalued part of $T_{\theta \times}$ (see Assertion 3.1);

(ii) $Y_{I}(\cdot, \lambda)(\in[\mathbb{H}])$ is an operator solution of $(3.1)$ with $Y_{I}(a, \lambda)=I_{\mathbb{H}}$ and $\mathfrak{H}_{1} \subset \mathfrak{H}$ is the set of all $\widetilde{f} \in \mathfrak{H}$ such that for some (and hence for all) $f(\cdot) \in \widetilde{f}$ the following relations hold:

$$
\begin{aligned}
\Delta(t) Y_{I}(t, 0) & J \int_{t}^{b} Y_{I}^{*}(u, 0) \Delta(u) f(u) d u=0 \quad(\text { a.e. on } \mathcal{I}), \\
& J \int_{\mathcal{I}} Y_{I}^{*}(t, 0) \Delta(t) f(t) d t \in \theta^{\times} .
\end{aligned}
$$

(iii) the assumption (A0) before (3.7) is fulfilled and

$$
\mathfrak{H}_{2}:=\left\{\tilde{f} \in \mathfrak{H}: \widehat{f}_{\widetilde{f}}(s)=0, \quad s \in \mathbb{R}\right\} .
$$

Then $\operatorname{mul} T_{\theta^{\times}}=\mathfrak{H}_{1}=\mathfrak{H}_{2}$. 
Proof. Let $\tilde{f} \in \mathfrak{H}$ and let

$$
y_{\widetilde{f}}=Y_{I}(t, 0) J \int_{t}^{b} Y_{I}^{*}(u, 0) \Delta(u) f(u) d u, \quad f(\cdot) \in \widetilde{f} .
$$

The immediate checking shows that $y_{\widetilde{f}}$ satisfies (3.5). Moreover, since $Y_{I}(\cdot, 0) \in \mathcal{L}_{\Delta}^{2}[\mathbb{H}]$, it follows that

$$
\begin{gathered}
\int_{\mathcal{I}}\left\|\Delta^{\frac{1}{2}}(t) y_{\widetilde{f}}(t)\right\|^{2} d t=\int_{\mathcal{I}}\left\|\Delta^{\frac{1}{2}}(t) Y_{I}(t, 0) J \int_{t}^{b} Y_{I}^{*}(u, 0) \Delta(u) f(u) d u\right\|^{2} d t \\
\leq\left(\int_{\mathcal{I}}\left\|Y_{I}^{*}(u, 0) \Delta(u) f(u)\right\| d u\right)^{2} \cdot \int_{\mathcal{I}}\left\|\Delta^{\frac{1}{2}}(t) Y_{I}(t, 0)\right\|^{2} d t<\infty .
\end{gathered}
$$

Hence $y_{\widetilde{f}} \in \mathcal{L}_{\Delta}^{2}(\mathcal{I} ; \mathbb{H})$ and, consequently, $y_{\widetilde{f}} \in \operatorname{dom} \mathcal{T}_{\max }$. Moreover, $\Gamma_{b} y_{\widetilde{f}}=0$, where $\Gamma_{b}$ is the operator (4.60) with $Y_{\widetilde{U}}=Y_{I}$. Therefore by (4.4) $\left[y_{\tilde{f}}, z\right]_{b}=0, z \in \operatorname{dom} \mathcal{T}_{\max }$. Assume now that $\bar{y}$ is a solution of (3.5) such that $\bar{y} \in \operatorname{dom} \mathcal{T}_{\max }$ and $[\bar{y}, z]_{b}=0, z \in \operatorname{dom} \mathcal{T}_{\max }$. Moreover, let $\widehat{y}=y_{\widetilde{f}}-\bar{y}$. Since $[\widehat{y}, z]_{b}=0, z \in \operatorname{dom} \mathcal{T}_{\max }$, it follows from (4.4) and surjectivity of $\Gamma_{b}$ that $\Gamma_{b} \widehat{y}=0$. On the other hand, $\widehat{y} \in \mathcal{N}_{0}$ and hence $\widehat{y}=Y_{I}(t, 0) h$ with some $h \in \mathbb{H}$. Moreover, by (4.60) $\Gamma_{b} \widehat{y}=h$. Therefore $h=0$, which implies $\widehat{y}=0$ and $y_{\widetilde{f}}=\bar{y}$. Thus $y_{\widetilde{f}}$ is a unique solution of (3.5) such that $y_{\tilde{f}} \in \operatorname{dom} \mathcal{T}_{\max }$ and $\left[y_{\tilde{f}}, z\right]_{b}=0, z \in \operatorname{dom} \mathcal{T}_{\text {max }}$. This statement and Assertion 3.1 yield the equality mul $T_{\theta^{\times}}=\mathfrak{H}_{1}$.

Next assume that $\tilde{f} \in \operatorname{mul} T_{\theta^{\times}}$and $f(\cdot) \in \widetilde{f}$. Then according to Assertion 3.1 there exists a function $y \in \operatorname{dom} \mathcal{T}_{\max }$ such that $\{y, f\} \in$ $\mathcal{T}_{\max }$ and (3.6) holds. For fixed $s \in \mathbb{R}$ and $h \in \mathbb{H}_{0}^{\prime}$ put $z=z(t):=$ $Y_{K}(t, s) h$. Then $\{z, s z\} \in \mathcal{T}_{\max }$ and application of the Lagrange's identity (3.3) to $\{y, f\}$ and $\{z, s z\}$ gives

$$
(f, z)_{\Delta}-s(y, z)_{\Delta}=[y, z]_{b}-(J y(a), z(a)) .
$$

Here

$$
\begin{gathered}
(f, z)_{\Delta}=\int_{\mathcal{I}}\left(\Delta(t) f(t), Y_{K}(t, s) h\right) d t \\
=\left(\int_{\mathcal{I}} Y_{K}^{*}(t, s) \Delta(t) f(t) d t, h\right)=\left(\widehat{f}_{\widetilde{f}}(s), h\right)
\end{gathered}
$$

and in view of the first equality in (3.6) one has $(y, z)_{\Delta}=0$. Moreover, by (3.6) $y(a) \in \theta^{\times}$and $z(a)=K h \in \theta$, which yields $(J y(a), z(a))=0$. Observe also that according to (3.6) $[y, z]_{b}=0$. Therefore (4.67) yields $\left(\widehat{f}_{\widetilde{f}}(s), h\right)=0, s \in \mathbb{R}, h \in \mathbb{H}_{0}^{\prime}$, and, consequently, $\widehat{f}_{\widetilde{f}}(s)=0, s \in \mathbb{R}$. Hence $\tilde{f} \in \mathfrak{H}_{2}$, which proves the inclusion mul $T_{\theta} \times \mathfrak{H}_{2}$. On the other hand, for each pseudospectral function $\sigma(\cdot)$ (with respect to $K_{\theta}$ ) one has $\mathfrak{H}_{2} \subset \operatorname{ker} V_{\sigma}=\operatorname{mul} T_{\theta^{\times}}$. Therefore the equality mul $T_{\theta^{\times}}=\mathfrak{H}_{2}$ is valid. 
Arguments just before Proposition 4.14 show that for a quasiregular system Definition 3.2 should be modified as follows.

Definition 4.15. Let system (3.1) be quasiregular, let $\theta$ be a subspace in $\mathbb{H}$ and let the assumption (A0) before (3.7) be fulfilled. A distribution function $\sigma(\cdot): \mathbb{R} \rightarrow\left[\mathbb{H}_{0}^{\prime}\right]$ is called a pseudospectral function of the system (with respect to the operator $\left.K=K_{\theta}\right)$ if $\widehat{f}=\widehat{f}_{\widetilde{f}} \in \mathcal{L}^{2}\left(\sigma ; \mathbb{H}_{0}^{\prime}\right)$ for all $\widetilde{f} \in \mathfrak{H}$ and the equality $V_{\sigma} \widetilde{f}=\pi_{\sigma} \widehat{f}_{\widetilde{f}}, \widetilde{f} \in \mathfrak{H}$, defines a partial isometry (the Fourier transform) $V_{\sigma} \in\left[\mathfrak{H}, L^{2}\left(\sigma ; \mathbb{H}_{0}^{\prime}\right)\right]$ with $\operatorname{ker} V_{\sigma}=\operatorname{mul} T_{\theta^{\times}}$.

If $V_{\sigma}$ is an isometry, then $\sigma(\cdot)$ is called a spectral function.

Remark 4.16. (1) As is known [22], each symmetric differential operator of an even order is reduced to a certain symmetric Hamiltonian system. For quasiregular differential operators of an even order an analog of the monodromy matrix $(4.61)$ was used in $[16,21]$ for parametrization of all Titchmarsh-Weyl functions. Observe also that formula similar to (4.13) was obtained in [18] for Stourm-Liouville operators with the operator valued potential.

(2) Let $\mathfrak{H}_{1}$ and $\mathfrak{H}_{2}$ be subspaces in $\mathfrak{H}$ defined in Proposition 4.14. Then by this proposition the condition $\operatorname{ker} V_{\sigma}=\operatorname{mul} T_{\theta^{\times}}$in Definition 4.15 of a pseudospectral function can be replaced with $\operatorname{ker} V_{\sigma}=\mathfrak{H}_{1}\left(=\mathfrak{H}_{2}\right)$. Therefore for the regular canonical Hamiltonian system (3.1) in the case $\theta=H \oplus\{0\}$ our definition of the pseudospectral function coincides with that introduced for such systems in [36]. Observe also that for such systems the equality $\mathfrak{H}_{1}=\mathfrak{H}_{2}$ is proved in [37, Lemma A.18].

\subsection{Special cases}

The results of the previous subsection are simplified in the following four special cases:

1. The case $\mathbb{H}_{0}=\mathbb{H}$. Let under the assumptions (B1)-(B3) from Section $4.1 \mathbb{H}_{0}=\mathbb{H}$. Then $H_{1}=H, H_{1}^{\perp}=\{0\}$ and the monodromy matrix $B(\cdot)$ admits the block representation

$$
B(\lambda)=\left(\begin{array}{lll}
B_{11}(\lambda) & B_{12}(\lambda) & B_{13}(\lambda) \\
B_{21}(\lambda) & B_{22}(\lambda) & B_{23}(\lambda) \\
B_{31}(\lambda) & B_{32}(\lambda) & B_{33}(\lambda)
\end{array}\right): H \oplus \widehat{H} \oplus H \rightarrow H \oplus \widehat{H} \oplus H
$$

Moreover, the operator function $W(\cdot)$ is defined by (4.9) with $\mathbb{H}$ instead of $\mathbb{H}_{0}$ and in view of (4.10)-(4.13) the entries $w_{j}(\lambda)$ are given in terms of 
entries $B_{i j}=B_{i j}(\lambda)$ from (4.68) by

$$
\begin{gathered}
w_{1}(\lambda)=\left(\begin{array}{ccc}
B_{11} & B_{12} & B_{13} \\
-i B_{21} & -i\left(B_{22}-I_{\widehat{H}}\right) & -i B_{23} \\
0 & 0 & -I_{H}
\end{array}\right): \underbrace{H \oplus \widehat{H} \oplus H}_{\mathbb{H}} \\
w_{2}(\lambda)=\left(\begin{array}{ccc}
\frac{1}{2} B_{13} & \frac{i}{2} B_{12} & -\frac{1}{2} B_{11} \\
-\frac{i}{2} B_{23} & \frac{1}{2}\left(B_{22}+I_{\widehat{H}}\right) & \frac{i}{2} B_{21} \\
\frac{1}{2} I_{H} & 0 & 0
\end{array}\right): H \oplus \widehat{H} \oplus H \\
w_{3}(\lambda)=\left(\begin{array}{ccc}
B_{31} & \rightarrow H \oplus \widehat{H} \oplus H \\
-\frac{1}{2} B_{21} & -\frac{1}{2}\left(B_{22}+I_{\widehat{H}}\right) & -\frac{1}{2} B_{23} \\
-I_{H} & 0 & 0
\end{array}\right): H \oplus \widehat{H} \oplus H \\
w_{4}(\lambda)=\left(\begin{array}{ccc}
\frac{1}{2} B_{33} & \rightarrow H \oplus \widehat{H} \oplus H \\
-\frac{1}{4} B_{23} & -\frac{i}{4}\left(B_{22}-I_{\widehat{H}}\right) & \frac{1}{4} B_{21} \\
0 & 0 & -\frac{1}{2} I_{H}
\end{array}\right): H \oplus \widehat{H} \oplus H
\end{gathered}
$$

Observe also that in this case $U=\widetilde{U}$ and the operator solutions $Y_{U}(\cdot, \lambda) \in$ $\mathcal{L}_{\Delta}^{2}[\mathbb{H}]$ and $\psi(\cdot, \lambda) \in \mathcal{L}_{\Delta}^{2}[\mathbb{H}]$ in Proposition 4.4 satisfies $\widetilde{U}^{-1} Y_{U}(a, \lambda)=I_{\mathbb{H}}$ (that is $\left.Y_{U}(\cdot, \lambda)=Y_{\widetilde{U}}(\cdot, \lambda)\right)$ and $\widetilde{U}^{-1} \psi(a, \lambda)=\frac{1}{2} J$.

2. The case $\mathbb{H}_{0}=H \oplus \widehat{H}$. Let under the assumptions (B1)-(B3) $\mathbb{H}_{0}=H \oplus \widehat{H}$ (this means that the subspace $\mathbb{H}_{0}$ is minimally possible). Then $H_{1}=\{0\}, H_{1}^{\perp}=H$ and hence the monodromy matrix $B(\cdot)$ admits the block representation (4.68). Moreover, in view of (4.10)-(4.13) the operator function $W(\cdot)$ is of the form (4.9) with entries $w_{j}(\lambda) \in[H \oplus \widehat{H}]$ defined in terms of entries $B_{i j}=B_{i j}(\lambda)$ from (4.68) by (1.8) and (1.9). Note also that in this case the initial conditions for operator solutions $Y_{U}(\cdot, \lambda)$ and $\psi(\cdot, \lambda)$ of $(3.1)$ (see Proposition 4.4) take the form

$$
\widetilde{U}^{-1} Y_{U}(a, \lambda)=\left(\begin{array}{cc}
I_{H} & 0 \\
0 & I_{\widehat{H}} \\
0 & 0
\end{array}\right), \quad \widetilde{U}^{-1} \psi(a, \lambda)=\left(\begin{array}{cc}
0 & 0 \\
0 & \frac{i}{2} I_{\widehat{H}} \\
I_{H} & 0
\end{array}\right),
$$

(the block operators in the right hand parts act from $H \oplus \widehat{H}$ to $H \oplus \widehat{H} \oplus H$ ).

3. The case of the Hamiltonian system. Recall that system (3.1) is called Hamiltonian if $\mathbb{H}=H \oplus H(\Leftrightarrow \widehat{H}=\{0\})$ and the operator $J$ is 
given by (1.3). For the Hamiltonian system the assumptions (B1)-(B3) from Section 4.1 takes the following form:

(HB1) The equality $N_{+}=n$ is valid.

(HB2) $\theta$ is a linear relation in $H$ such that $\theta^{*}$ is a symmetric relation in $H$. Moreover, $H_{1}$ is a subspace in $H, H_{1}^{\perp}=H \ominus H_{1}$,

$$
\mathbb{H}_{0}=H \oplus H_{1}=H_{1}^{\perp} \oplus H_{1} \oplus H_{1}, \quad \mathbb{H}_{0} \subset \mathbb{H},
$$

and $\widetilde{U} \in[\mathbb{H}]$ is an operator such that $\widetilde{U}^{*} J \widetilde{U}=J$ and $\widetilde{U} \mathbb{H}_{0}=\theta$

(HB3) $H^{\prime}$ is a subspace in $H$ and $\Gamma_{b}=\left(\begin{array}{l}\Gamma_{0 b} \\ \Gamma_{1 b}\end{array}\right): \operatorname{dom} \mathcal{T}_{\max } \rightarrow H \oplus H$ is the operator such that

$$
\begin{gathered}
P_{H, H^{\prime}} \Gamma_{1 b}=-\frac{i}{2} P_{H, H^{\prime}} \Gamma_{0 b} \\
\operatorname{ran} \Gamma_{b}=\left\{h \oplus h^{\prime} \in H \oplus H: P_{H, H^{\prime}} h^{\prime}=-\frac{i}{2} P_{H, H^{\prime}} h\right\} \\
{[y, z]_{b}=\left(J \Gamma_{b} y, \Gamma_{b} z\right)=\left(\Gamma_{0 b} y, \Gamma_{1 b} z\right)-\left(\Gamma_{1 b} y, \Gamma_{0 b} z\right), \quad y, z \in \operatorname{dom} \mathcal{T}_{\max } .}
\end{gathered}
$$

If the Hamiltonian system (3.1) satisfies the assumptions (HB1)-(HB3), then the monodromy matrix $B(\cdot)$ admits the block representation

$$
\begin{aligned}
& B(\lambda)=\left(\begin{array}{llll}
B_{11}(\lambda) & B_{12}(\lambda) & B_{13}(\lambda) & B_{14}(\lambda) \\
B_{21}(\lambda) & B_{22}(\lambda) & B_{23}(\lambda) & B_{24}(\lambda)
\end{array}\right): \underbrace{H_{1}^{\perp} \oplus H_{1}}_{H} \oplus \underbrace{H_{1} \oplus H_{1}^{\perp}}_{H} \\
& \rightarrow H \oplus H
\end{aligned}
$$

and (4.10)-(4.13) imply that the entries $w_{j}(\lambda)$ in (4.9) are defined by

$$
\begin{gathered}
w_{1}(\lambda)=\left(\begin{array}{ccc}
B_{11}(\lambda) & B_{12}(\lambda) & B_{13}(\lambda) \\
0 & 0 & -I_{H_{1}}
\end{array}\right): \underbrace{H_{1}^{\perp} \oplus H_{1} \oplus H_{1}}_{\mathbb{H}_{0}} \rightarrow \underbrace{H \oplus H_{1}}_{\mathbb{H}_{0}} \\
w_{2}(\lambda)=\left(\begin{array}{ccc}
B_{14}(\lambda) & \frac{1}{2} B_{13}(\lambda) & -\frac{1}{2} B_{12}(\lambda) \\
0 & \frac{1}{2} I_{H_{1}} & 0
\end{array}\right): H_{1}^{\perp} \oplus H_{1} \oplus H_{1} \rightarrow H \oplus H_{1} \\
w_{3}(\lambda)=\left(\begin{array}{ccc}
B_{21}(\lambda) & B_{22}(\lambda) & B_{23}(\lambda) \\
0 & -I_{H_{1}} & 0
\end{array}\right): H_{1}^{\perp} \oplus H_{1} \oplus H_{1} \rightarrow H \oplus H_{1} \\
w_{4}(\lambda)=\left(\begin{array}{ccc}
B_{24}(\lambda) & \frac{1}{2} B_{23}(\lambda) & -\frac{1}{2} B_{22}(\lambda) \\
0 & 0 & -\frac{1}{2} I_{H_{1}}
\end{array}\right): H_{1}^{\perp} \oplus H_{1} \oplus H_{1} \rightarrow H \oplus H_{1}
\end{gathered}
$$

(here $B_{i j}(\lambda)$ are taken from (4.3)). If in addition $\mathbb{H}_{0}=\mathbb{H}$, then $H_{1}^{\perp}=$ $\{0\}, H_{1}=H$ and $B(\cdot)$ admits the block representation

$$
B(\lambda)=\left(\begin{array}{ll}
B_{11}(\lambda) & B_{12}(\lambda) \\
B_{21}(\lambda) & B_{22}(\lambda)
\end{array}\right): H \oplus H \rightarrow H \oplus H .
$$


In this case

$$
\begin{gathered}
w_{1}(\lambda)=\left(\begin{array}{cc}
B_{11} & B_{12} \\
0 & -I_{H}
\end{array}\right)(\in[H \oplus H]), \\
w_{2}(\lambda)=\left(\begin{array}{cc}
\frac{1}{2} B_{12} & -\frac{1}{2} B_{11} \\
\frac{1}{2} I_{H} & 0
\end{array}\right)(\in[H \oplus H]), \\
w_{3}(\lambda)=\left(\begin{array}{cc}
B_{21} & B_{22} \\
-I_{H} & 0
\end{array}\right)(\in[H \oplus H]), \\
w_{4}(\lambda)=\left(\begin{array}{cc}
\frac{1}{2} B_{22} & -\frac{1}{2} B_{21} \\
0 & -\frac{1}{2} I_{H}
\end{array}\right)(\in[H \oplus H]),
\end{gathered}
$$

where $B_{i j}=B_{i j}(\lambda)$ are taken from (4.78).

The simplest situation takes place when $\mathbb{H}_{0}=H$. In this case $H_{1}=$ $\{0\}, H_{1}^{\perp}=H$ and $B(\cdot)$ admits the representation (4.78). Moreover,

$$
W(\lambda)=B(\lambda)=\left(\begin{array}{ll}
B_{11}(\lambda) & B_{12}(\lambda) \\
B_{21}(\lambda) & B_{22}(\lambda)
\end{array}\right): H \oplus H \rightarrow H \oplus H,
$$

that is the operator function $W(\cdot)$ coincides with the monodromy matrix $B(\cdot)$. Observe also that in this case the operator solutions $Y_{U}(\cdot, \lambda) \in$ $\mathcal{L}_{\Delta}^{2}[H, H \oplus H]$ and $\psi(\cdot, \lambda) \in \mathcal{L}_{\Delta}^{2}[H, H \oplus H]$ of (3.1) (see Proposition 4.4) are defined by the initial values (4.13).

4. The case of the differential equation of the first order. Assume that $H=\{0\}$. Then $\mathbb{H}=\widehat{H}, J=i I_{\mathbb{H}}$ and system (3.1) takes the form of the first order differential equation

$$
i y^{\prime}-A(t) y=\lambda \Delta(t) y, \quad t \in \mathcal{I}=[a, b\rangle, \quad \lambda \in \mathbb{C}
$$

with operator-valued coefficients $A(t), B(t)(\in[\mathbb{H}])$. Moreover, by $[22$, Theorem 2.2] for this system $N_{-}=n(=\operatorname{dim} \mathbb{H})$.

Assume now that $N_{+}=N_{-}=n$, that is system (4.82) is quasiregular. Since $H=\{0\}$, the class $\operatorname{Sym}(\mathbb{H})$ consists only of the trivial relation $\theta=\{0\}$. This and Remark 4.10 imply that the assumptions (B2) and (B3) in Section 4.1 take the form:

(B2') $\theta=\mathbb{H}$ and $\widetilde{U} \in[\mathbb{H}]$ is a unitary operator.

(B3') $\Gamma_{b}: \operatorname{dom} \mathcal{T}_{\max } \rightarrow \mathbb{H}$ is a surjective operator satisfying

$$
[y, z]_{b}=i\left(\Gamma_{b} y, \Gamma_{b} z\right), \quad y, z \in \operatorname{dom} \mathcal{T}_{\max } .
$$

Moreover, the monodromy matrix $B(\lambda)$ is defined by (4.6) and the operator-function $W(\cdot)$ (see (4.9)) is

$$
W(\lambda)=\left(\begin{array}{ll}
w_{1}(\lambda) & w_{2}(\lambda) \\
w_{3}(\lambda) & w_{4}(\lambda)
\end{array}\right)=\left(\begin{array}{cc}
-i(B(\lambda)-I) & \frac{1}{2}(B(\lambda)+I) \\
-\frac{1}{2}(B(\lambda)+I) & -\frac{i}{4}(B(\lambda)-I)
\end{array}\right) \in[\mathbb{H} \oplus \mathbb{H}] .
$$


By using (4.84) and (4.43) one can easily prove that for definite system (4.82) $\|B(\lambda) h\| \geq \alpha_{\lambda}\|h\|, h \in \mathbb{H}$, with some $\alpha_{\lambda}>1, \lambda \in \mathbb{C}_{+}$.

\subsection{Parametrization of pseudospectral functions}

A parametrization of all pseudospectral and spectral functions of the symmetric system with the maximal deficiency index $N_{+}=n$ is given in the following theorem.

Theorem 4.17. Let for system (3.1) the assumptions (B1)-(B3) in Section 4.1 be satisfied, let $W(\cdot)$ be the operator function (4.9)-(4.13) and let $\mathbb{H}_{1}$ be a subspace in $\mathbb{H}_{0}$ given by

$$
\mathbb{H}_{1}=\mathcal{H}_{b} \oplus \widehat{H} \oplus H_{1}
$$

with $\mathcal{H}_{b}=H \ominus H^{\prime}$. Assume also that the system is $\theta$-definite. Then:

(1) The equality

$$
\begin{aligned}
& m_{\widetilde{\tau}}(\lambda)=\left(\widetilde{C}_{0}(\lambda) w_{1}(\lambda)+\widetilde{C}_{1}(\lambda) w_{3}(\lambda)\right)^{-1} \\
& \times\left(\widetilde{C}_{0}(\lambda) w_{2}(\lambda)+\widetilde{C}_{1}(\lambda) w_{4}(\lambda)\right), \quad \lambda \in \mathbb{C}_{+}
\end{aligned}
$$

together with the Stieltjes inversion formula (3.29) establishes a bijective correspondence $\sigma(s)=\sigma_{\widetilde{\tau}}(s)$ between all pairs

$$
\widetilde{\tau}=\left\{\widetilde{C}_{0}(\lambda), \widetilde{C}_{1}(\lambda)\right\} \in \widetilde{R}_{\mathbb{H}_{1}}\left(\mathbb{H}_{0}\right)
$$

of operator functions $\widetilde{C}_{j}(\cdot): \mathbb{C}_{+} \rightarrow\left[\mathbb{H}_{0}\right], j \in\{0,1\}$, satisfying

$$
\begin{aligned}
& \lim _{y \rightarrow+\infty} \frac{1}{i y} P_{\mathbb{H}_{0}, \mathbb{H}_{1}} w_{1}(i y)\left(\widetilde{C}_{0}(i y) w_{1}(i y)+\widetilde{C}_{1}(i y) w_{3}(i y)\right)^{-1} \widetilde{C}_{1}(i y) \uparrow \mathbb{H}_{1}=0 \\
& \lim _{y \rightarrow+\infty} \frac{1}{i y} P_{\mathbb{H}_{0}, \mathbb{H}_{1}} w_{3}(i y)\left(\widetilde{C}_{0}(i y) w_{1}(i y)+\widetilde{C}_{1}(i y) w_{3}(i y)\right)^{-1} \widetilde{C}_{0}(i y) \uparrow \mathbb{H}_{1}=0
\end{aligned}
$$

and all $\mathbb{H}_{0}$-valued pseudospectral functions $\sigma(\cdot)$ (with respect to the operator $U$ of the form (3.27)). If in addition the system is quasiregular, then in (4.87) $\widetilde{R}_{\mathbb{H}_{1}}\left(\mathbb{H}_{0}\right)=\widetilde{R}\left(\mathbb{H}_{0}\right)$ and the conditions (4.88) and (4.89) take the form

$$
\begin{aligned}
& \lim _{y \rightarrow+\infty} \frac{1}{i y} w_{1}(i y)\left(\widetilde{C}_{0}(i y) w_{1}(i y)+\widetilde{C}_{1}(i y) w_{3}(i y)\right)^{-1} \widetilde{C}_{1}(i y)=0 \\
& \lim _{y \rightarrow+\infty} \frac{1}{i y} w_{3}(i y)\left(\widetilde{C}_{0}(i y) w_{1}(i y)+\widetilde{C}_{1}(i y) w_{3}(i y)\right)^{-1} \widetilde{C}_{0}(i y)=0,
\end{aligned}
$$

In this case $V_{\sigma} \mathfrak{H}=L^{2}\left(\sigma ; \mathbb{H}_{0}\right)$ if and only if $\widetilde{\tau} \in \widetilde{R}^{0}\left(\mathbb{H}_{0}\right)$. 
(2) Condition (4.89) in statement (1) can be omitted if and only if

$$
\lim _{y \rightarrow+\infty} \frac{1}{i y} w_{3}(i y) w_{1}^{-1}(i y)=0
$$

(3) The following statements are equivalent:

(a) all pairs (4.87) satisfy (4.88) and (4.89);

(b) statement (1) is valid for an arbitrary pair (4.87);

(c) $\operatorname{mul} T_{\theta^{\times}}=\operatorname{mul} T_{\theta^{\times}}^{*}$

(d) $\lim _{y \rightarrow+\infty} \frac{1}{i y} w_{3}(i y) w_{1}^{-1}(i y)=0$ and $\lim _{y \rightarrow+\infty} y \cdot \operatorname{Im}\left(w_{3}(i y) w_{1}^{-1}(i y) h, h\right)=$ $-\infty, \quad 0 \neq h \in \mathbb{H}$.

(e) for some (and hence any) fixed $\lambda_{0} \in \mathbb{C}_{+}$the operator function

$$
\chi(\lambda)=\left(w_{3}(\lambda)+\lambda_{0} w_{1}(\lambda)\right)\left(w_{3}(\lambda)-\lambda_{0} w_{1}(\lambda)\right)^{-1}, \quad \lambda \in \mathbb{C}_{+} .
$$

satisfies the condition

$$
\lim _{y \rightarrow+\infty} y(\|h\|-\|\chi(i y) h\|)=+\infty, \quad 0 \neq h \in \mathbb{H}_{0} .
$$

(4) If in addition mul $T_{\theta^{\times}}=\{0\}$, then statements (1) and (2) are valid for spectral functions (instead of pseudospectral ones). For quasiregular systems the condition mul $T_{\theta^{\times}}=\{0\}$ is equivalent to $\mathfrak{H}_{1}\left(=\mathfrak{H}_{2}\right)=\{0\}$, where $\mathfrak{H}_{1}$ and $\mathfrak{H}_{2}$ are defined in Proposition 4.14.

Proof. (1) Let $\dot{\mathcal{H}}_{0}$ and $\dot{\mathcal{H}}_{1}$ be given by (4.15). Then according to arguments before Proposition 4.3 the assumptions (A1)-(A3) are satisfied (with $\Gamma_{b}^{\prime}$ of the form (4.14)) and hence the equalities (3.20)-(3.23) define the operator functions $m_{0}, S_{1}, S_{2}$ and $\dot{\mathcal{M}}$. Moreover, $W(\lambda)$ admits the representation (4.3) and the standard calculations (see e.g. [34, Theorem 6.16]) imply that for each boundary parameter $\bar{\tau}=\left\{\bar{C}_{0}(\lambda), \bar{C}_{1}(\lambda)\right\} \in$ $\widetilde{R}_{\dot{\mathcal{H}}_{1}}\left(\dot{\mathcal{H}}_{0}\right)$ the equality $(3.28)$ can be written as

$$
\begin{aligned}
m_{\bar{\tau}}(\lambda)=\left(\widetilde{C}_{0}(\lambda) w_{1}(\lambda)+\right. & \left.\widetilde{C}_{1}(\lambda) w_{3}(\lambda)\right)^{-1} \\
& \left(\widetilde{C}_{0}(\lambda) w_{2}(\lambda)+\widetilde{C}_{1}(\lambda) w_{4}(\lambda)\right), \quad \lambda \in \mathbb{C}_{+} .
\end{aligned}
$$

with

$$
\widetilde{C}_{0}(\lambda)=U_{0} \bar{C}_{0}(\lambda) U_{0}^{-1}, \quad \widetilde{C}_{1}(\lambda)=U_{0} \bar{C}_{1}(\lambda) U_{0}^{-1}, \quad \lambda \in \mathbb{C}_{+}
$$

(here $U_{0}$ is given by (4.17)). Since $U_{0} \dot{\mathcal{H}}_{1}=\mathbb{H}_{1}$, the equalities (4.95) give a bijective correspondence between all boundary parameters $\bar{\tau}$ and all pairs $\widetilde{\tau}$ of the form (4.87). Moreover, since by (4.3)

$$
\dot{\mathcal{M}}(\lambda)=-U_{0}^{-1} w_{3}(\lambda) w_{1}^{-1}(\lambda) U_{0},
$$


it follows that

$$
\left(\bar{C}_{0}(\lambda)-\bar{C}_{1}(\lambda) \dot{\mathcal{M}}(\lambda)\right)^{-1}=U_{0}^{-1} w_{1}(\lambda)\left(\widetilde{C}_{0}(\lambda) w_{1}(\lambda)+\widetilde{C}_{1}(\lambda) w_{3}(\lambda)\right)^{-1} U_{0}
$$

Therefore

$$
\begin{gathered}
P_{\dot{\mathcal{H}}_{0}, \dot{\mathcal{H}}_{1}}\left(\bar{C}_{0}(\lambda)-\bar{C}_{1}(\lambda) \dot{\mathcal{M}}(\lambda)\right)^{-1} \bar{C}_{1}(\lambda) \uparrow \dot{\mathcal{H}}_{1} \\
=U_{01}^{-1} P_{\mathbb{H}_{0}, \mathbb{H}_{1}} w_{1}(\lambda)\left(\widetilde{C}_{0}(\lambda) w_{1}(\lambda)+\widetilde{C}_{1}(\lambda) w_{3}(\lambda)\right)^{-1} \widetilde{C}_{1}(\lambda) \uparrow \mathbb{H}_{1} \cdot U_{01} \\
P_{\dot{\mathcal{H}}_{0}, \dot{\mathcal{H}}_{1}} \dot{\mathcal{M}}(\lambda)\left(\bar{C}_{0}(\lambda)-\bar{C}_{1}(\lambda) \dot{\mathcal{M}}(\lambda)\right)^{-1} \bar{C}_{0}(\lambda) \uparrow \dot{\mathcal{H}}_{1} \\
=-U_{01}^{-1} P_{\mathbb{H}_{0}, \mathbb{H}_{1}} w_{3}(\lambda)\left(\widetilde{C}_{0}(\lambda) w_{1}(\lambda)+\widetilde{C}_{1}(\lambda) w_{3}(\lambda)\right)^{-1} \widetilde{C}_{0}(\lambda) \uparrow \mathbb{H}_{1} \cdot U_{01}
\end{gathered}
$$

with $U_{01}=U_{0} \uparrow \dot{\mathcal{H}}_{1} \in\left[\dot{\mathcal{H}}_{1}, \mathbb{H}_{1}\right]$ and hence the boundary parameter $\bar{\tau}$ is admissible in the sense of Definition 3.11 if and only if the pair $\widetilde{\tau}$ satisfies (4.88) and (4.89). Now the required correspondence between pairs $\widetilde{\tau}$ and pseudospectral functions is implied by Theorem 3.12, (1).

If the system is quasiregular, then by (4.5) $H^{\prime}=\{0\}$ and hence $\mathcal{H}_{b}=H, \mathbb{H}_{1}=\mathbb{H}_{0}$. Therefore $\widetilde{R}_{\mathbb{H}_{1}}\left(\mathbb{H}_{0}\right)=\widetilde{R}\left(\mathbb{H}_{0}\right)$ and the conditions (4.88), (4.89) take the form (4.90), (4.91).

Statement (2) follows from (4.96), (4.97) and Lemma 3.14.

(3) It follows from (4.96) that statement (d) is equivalent to statement (d) of Theorem 3.12, (2). Therefore the equivalences (a) $\Leftrightarrow(\mathrm{b}) \Leftrightarrow(\mathrm{c}) \Leftrightarrow$ (d) are consequences of this theorem. Next, in view of (4.96) the operator function (2.1) for $\dot{\mathcal{M}}(\lambda) \in R_{u}\left[\dot{\mathcal{H}}_{0}\right]$ is

$$
\begin{gathered}
C(\lambda)=\left(-U_{0}^{-1} w_{3}(\lambda) w_{1}^{-1}(\lambda) U_{0}-\lambda_{0} I\right)\left(-U_{0}^{-1} w_{3}(\lambda) w_{1}^{-1}(\lambda) U_{0}+\lambda_{0} I\right)^{-1} \\
=U_{0}^{-1} \chi(\lambda) U_{0}
\end{gathered}
$$

where $\chi(\lambda)$ is given by (4.92). Now applying Lemma 2.1 to $\dot{\mathcal{M}}(\lambda)$ one gets the equivalence $(\mathrm{d}) \Leftrightarrow(\mathrm{e})$.

(4) The first assertion follows from Proposition 3.4, while the second one is implied by Proposition 4.14.

Remark 4.18. (1) Assume that for system (3.1) $N_{+}=n, \theta$ is a subspace in $\mathbb{H}$ such that $\theta^{\times} \in \operatorname{Sym}(\mathbb{H})$ and the assumption (A0) before Definition 3.2 is fulfilled. It follows from [35, Proposition 3.12] that in this case Theorem 4.17 remains valid (with some obvious modifications) for pseudospectral and spectral functions with respect to $K_{\theta}$ in place of $U$.

(2) In the extremal cases $\mathbb{H}_{0}=\mathbb{H}$ and $\mathbb{H}_{0}=H \oplus \widehat{H}$ the parametrisation of $\left[\mathbb{H}_{0}\right]$-valued pseudospectral functions is given by Theorem 4.17 with coefficients $w_{j}(\lambda)$ in (4.17) defined by (4.69)-(4.72) and (1.8), (1.9) respectively. 
(3) Theorem 4.17 and identity (4.46) show that the operator-function $W(\lambda)$ is an analog of the Nevanlinna matrix in the moment problem [1] and the resolvent matrix in the extension theory of symmetric operators [23].

In the following theorem we parameterize all pseudospectral functions of the first order differential equation.

Theorem 4.19. Assume that system (differential equation of the first order) (4.82) is quasiregular and definite. Let $\Gamma_{b}: \operatorname{dom} \mathcal{T}_{\max } \rightarrow \mathbb{H}$ be a surjective operator satisfying (4.83), let $\widetilde{U} \in[\mathbb{H}]$ be a unitary operator and let $B(\cdot)$ be the monodromy matrix (4.6) (for the regular system one can put $\left.B(\lambda)=Y_{\widetilde{U}}(b, \lambda), \lambda \in \mathbb{C}\right)$. Then:

(1) The equalities

$$
\begin{array}{r}
m_{K}(\lambda)=\frac{i}{2}(B(\lambda)-K(\lambda))^{-1}(B(\lambda)+K(\lambda)), \quad \lambda \in \mathbb{C}_{+} \\
\sigma_{K}(s)=\lim _{\delta \rightarrow+0} \lim _{y \rightarrow+0} \frac{1}{\pi} \int_{-\delta}^{s-\delta} \operatorname{Im} m_{K}(x+i y) d x
\end{array}
$$

establish a bijective correspondence $\sigma(s)=\sigma_{K}(s)$ between all operatorfunctions $K(\cdot) \in \mathbf{C}[\mathbb{H}]$ satisfying

$$
\begin{aligned}
\lim _{y \rightarrow+\infty} \frac{1}{y}\left(B(i y)-I_{\mathbb{H}}\right)(B(i y)-K(i y))^{-1}\left(K(i y)-I_{\mathbb{H}}\right) & =0 \\
\lim _{y \rightarrow+\infty} \frac{1}{y}\left(B(i y)+I_{\mathbb{H}}\right)(B(i y)-K(i y))^{-1}\left(K(i y)+I_{\mathbb{H}}\right) & =0
\end{aligned}
$$

and all $[\mathbb{H}]$-valued pseudospectral functions $\sigma(\cdot)$ (with respect to the operator $\widetilde{U})$. Moreover, the admissibility conditions (4.100) and (4.101) can be omitted if and only if $\operatorname{mul} T_{\min }=\operatorname{mul} T_{\max }$ or, equivalently, if and only if

$$
\lim _{y \rightarrow+\infty} y(\|B(i y) h\|-\|h\|)=+\infty, \quad 0 \neq h \in \mathbb{H}_{0} .
$$

(2) If in addition mul $T_{\min }=\{0\}$, then statement (1) is valid for spectral functions (instead of pseudospectral ones).

Proof. It follows from Proposition 2.6 that the equalities

$$
\widetilde{C}_{0}(\lambda)=i\left(N(\lambda)+I_{\mathbb{H}}\right), \quad \widetilde{C}_{1}(\lambda)=N(\lambda)-I_{\mathbb{H}}, \quad \lambda \in \mathbb{C}_{+}
$$

give a bijective correspondence between all functions $N(\cdot) \in \mathbf{C}[\mathbb{H}]$ and all pairs $\widetilde{\tau}(\lambda)=\left\{\widetilde{C}_{0}(\lambda), \widetilde{C}_{1}(\lambda)\right\} \in \widetilde{R}(\mathbb{H})$. Moreover, (4.103) and (4.84) 
yield

$$
\begin{gathered}
\widetilde{C}_{0}(\lambda) w_{1}(\lambda)+\widetilde{C}_{1}(\lambda) w_{3}(\lambda) \\
=(N(\lambda)+I)(B(\lambda)-I)-\frac{1}{2}(N(\lambda)-I)(B(\lambda)+I) \\
=\frac{1}{2}((N(\lambda)+3 I) B(\lambda)-(3 N(\lambda)+I))=\frac{1}{2}(N(\lambda)+3 I)(B(\lambda)-K(\lambda)) ; \\
\left(\widetilde{C}_{0}(\lambda) w_{1}(\lambda)+\widetilde{C}_{1}(\lambda) w_{3}(\lambda)\right)^{-1}=2(B(\lambda)-K(\lambda))^{-1}(N(\lambda)+3 I)^{-1} ; \\
\widetilde{C}_{0}(\lambda) w_{2}(\lambda)+\widetilde{C}_{1}(\lambda) w_{4}(\lambda) \\
=\frac{i}{2}(N(\lambda)+I)(B(\lambda)+I)-\frac{i}{4}(N(\lambda)-I)(B(\lambda)-I) \\
=\frac{i}{4}((N(\lambda)+3 I) B(\lambda)+(3 N(\lambda)+I))=\frac{i}{4}(N(\lambda)+3 I)(B(\lambda)+K(\lambda)),
\end{gathered}
$$

where

$$
\begin{aligned}
& K(\lambda)=(N(\lambda)+3 I)^{-1}(3 N(\lambda)+I) \\
= & (3 N(\lambda)+I)(N(\lambda)+3 I)^{-1}, \quad \lambda \in \mathbb{C}_{+} .
\end{aligned}
$$

Therefore the operator-function $m_{K}(\lambda):=m_{\widetilde{\tau}}(\lambda)$ defined by (4.17) admits the representation (4.98) with $K(\lambda)$ of the form (4.4).

Let $X, J_{\mathbb{H}} \in[\mathbb{H} \oplus \mathbb{H}]$ be the operators defined by

$$
X=\frac{1}{\sqrt{8}}\left(\begin{array}{cc}
3 I_{\mathbb{H}} & I_{\mathbb{H}} \\
I_{\mathbb{H}} & 3 I_{\mathbb{H}}
\end{array}\right), \quad J_{\mathbb{H}}=\left(\begin{array}{cc}
I_{\mathbb{H}} & 0 \\
0 & -I_{\mathbb{H}}
\end{array}\right) .
$$

Then $X^{*} J_{\mathbb{H}} X=J_{\mathbb{H}}$ and according to [24] the equality (4.4) gives a bijection $N(\lambda) \rightarrow K(\lambda)$ of the set $\mathbf{C}[\mathbb{H}]$ onto itself. Moreover, the inverse bijection $K(\lambda) \rightarrow N(\lambda)$ is

$$
N(\lambda)=(K(\lambda)-3 I)^{-1}(I-3 K(\lambda)), \quad \lambda \in \mathbb{C}_{+} .
$$

and the following equalities hold

$$
\begin{gathered}
(N(\lambda)+3 I)^{-1}=[I-3 K(\lambda)+3(K(\lambda)-3 I)]^{-1}(K(\lambda)-3 I) \\
=-\frac{1}{8}(K(\lambda)-3 I) \\
N(\lambda)+I=-2(K(\lambda)-3 I)^{-1}(K(\lambda)+I), \\
N(\lambda)-I=-4(K(\lambda)-3 I)^{-1}(K(\lambda)-I) .
\end{gathered}
$$

Combining these equalities with (4.84), (4.4) and (4.103) one gets

$$
\begin{aligned}
& w_{1}(\lambda)\left(\widetilde{C}_{0}(\lambda) w_{1}(\lambda)+\widetilde{C}_{1}(\lambda) w_{3}(\lambda)\right)^{-1} \widetilde{C}_{1}(\lambda) \\
= & -i(B(\lambda)-I)(B(\lambda)-K(\lambda))^{-1}(K(\lambda)-I) \\
& w_{3}(\lambda)\left(\widetilde{C}_{0}(\lambda) w_{1}(\lambda)+\widetilde{C}_{1}(\lambda) w_{3}(\lambda)\right)^{-1} \widetilde{C}_{0}(\lambda) \\
= & -\frac{i}{4}(B(\lambda)+I)(B(\lambda)-K(\lambda))^{-1}(K(\lambda)+I) .
\end{aligned}
$$


Therefore the conditions (4.90) and (4.91) admit the representations (4.100) and (4.101).

Next, in view of (4.84) for $\lambda_{0}=\frac{i}{2}$ one has $\chi(\lambda)=B^{-1}(\lambda)$, where $\chi(\lambda)$ is given by (4.92). Now the required statements follow from Theorem 4.17 .

\section{References}

[1] N. I. Akhiezer, The classical moment problem, Oliver and Boyd, Edinburgh and London, 1965.

[2] S. Albeverio, M. M. Malamud, and V. I. Mogilevskii, On Titchmarsh-Weyl functions and eigenfunction expansions of first-order symmetric systems // Integr. Equ. Oper. Theory, 77 (2013), 303-354.

[3] D. Z. Arov, H. Dym, The bitangential inverse spectral problem for canonical systems // J. Fanct. Anal., 214 (2004), 312-385.

[4] D. Z. Arov, H. Dym, J-Contractive matrix valued functions and related topics, Encyclopedia of mathematics and its applications, Cambridge University Press, Cambridge, 2008.

[5] D. Z. Arov, H. Dym, Bitangential direct and inverse problems for systems of integral and differential equations, Encyclopedia of mathematics and its applications, Cambridge University Press, Cambridge, 2012.

[6] F. V. Atkinson, Discrete and continuous boundary problems, Academic Press, New York, 1963.

[7] J. Behrndt, S. Hassi, H. de Snoo, and R. Wiestma, Square-integrable solutions and Weyl functions for singular canonical systems // Math. Nachr., 284 (2011), No. 11-12, 1334-1383.

[8] V. M. Bruk, Extensions of symmetric relations // Math. Notes, 22 (1977), No.6, 953-958.

[9] V. A. Derkach, S. Hassi, M. M. Malamud, and H. S. V. de Snoo, Generalized resolvents of symmetric operators and admissibility // Methods of Functional Analysis and Topology, 6 (2000), No. 3 , 24-55.

[10] V. A. Derkach, S. Hassi, M. M. Malamud, and H. S. V. de Snoo, Boundary relations and generalized resolvents of symmetric operators // Russian J. Math. Ph., 16 (2009), No. 1, 17-60.

[11] V. A. Derkach, M. M. Malamud, Generalized resolvents and the boundary value problems for Hermitian operators with gaps // J. Funct. Anal., 95 (1991), 1-95.

[12] V. A. Derkach, M. M. Malamud, The extension theory of Hermitian operators and the moment problem // J. Math. Sciences, 73 (1995), No. 2, 141-242.

[13] A. Dijksma, H. Langer, and H.S.V. de Snoo, Hamiltonian systems with eigenvalue depending boundary conditions // Oper. Theory Adv. Appl., 35 (1988), 37-83.

[14] A. Dijksma, H. Langer, and H.S.V. de Snoo, Eigenvalues and pole functions of Hamiltonian systems with eigenvalue depending boundary conditions // Math. Nachr., 161 (1993), 107-153.

[15] N. Dunford, J. T. Schwartz, Linear operators. Part2. Spectral theory, Interscience Publishers, New York-London, 1963. 
[16] Ch. T. Fulton, Parametrizations of Titchmarsh's $m(\lambda)$-functions in the limit circle case // Trans. Amer. Math. Soc., 229 (1977), 51-63.

[17] I. Gohberg, M.G. Krein, Theory and applications of Volterra operators in Hilbert space // Transl. Math. Monographs, 24, Amer. Math. Soc., Providence, R.I., 1970.

[18] M. L. Gorbachuk, On spectral functios of a differential equation of the second order with operator-valued coefficients // Ukrain. Mat. Zh., 18 (1966), No. 2, $3-21$.

[19] V. I. Gorbachuk, M. L. Gorbachuk, Boundary problems for differential-operator equations, Kluver Acad. Publ., Dordrecht-Boston-London, 1991.

[20] I. S. Kats, Linear relations generated by the canonical differential equation of phase dimension 2, and eigenfunction expansion // St. Petersburg Math. J., 14 (2003), 429-452.

[21] A. M. Khol'kin, Description of selfadjoint extensions of differential operators of an arbitrary order on the infinite interval in the absolutely indefinite case // Teor. Funkcii Funkcional. Anal. Prilozhen., 44 (1985), 112-122.

[22] V. I. Kogan, F. S. Rofe-Beketov, On square-integrable solutions of symmetric systems of differential equations of arbitrary order // Proc. Roy. Soc. Edinburgh Sect. A, 74 (1974/75), 5-40.

[23] M. G. Krein, Sh. N. Saakyan, Some new results in the theory of resolvents of Hermitian operators // Soviet Math. Dokl., 7 (1966), 1086-1089.

[24] M. G. Krein, Ju. L. Shmul'jan, On linear-fractional transformations with operator coefficients // Amer. Math. Soc. Transl., 8 (1974), No. 2, 125-152; transl. Mat. Issled.2 (1967), No. 3, 34-96.

[25] H. Langer, B. Textorius, Spectral functions of a symmetric linear relation with a directing mapping, I // Proc. Roy. Soc. Edinburgh Sect. A, 97 (1984), 165176.

[26] H. Langer, B. Textorius, Spectral functions of a symmetric linear relation with a directing mapping, II // Proc. Roy. Soc. Edinburgh Sect. A, 101 (1985), $111-124$.

[27] M. Lesch, M. M. Malamud, On the deficiency indices and self-adjointness of symmetric Hamiltonian systems // J. Differential Equations, 189 (2003), 556615.

[28] M. M. Malamud, On the formula of generalized resolvents of a nondensely defined Hermitian operator // Ukr. Math. Zh., 44(1992), No. 12, 1658-1688.

[29] V. I. Mogilevskii, Boundary triplets and Krein type resolvent formula for symmetric operators with unequal defect numbers // Methods Funct. Anal. Topology, 12 (2006), No. 3, 258-280.

[30] V. I. Mogilevskii, Boundary pairs and boundary conditions for general (not necessarily definite) first-order symmetric systems with arbitrary deficiency indices // Math. Nachr., 285 (2012), No. 14-15, 1895-1931.

[31] V. I. Mogilevskii, On exit space extensions of symmetric operators with applications to first order symmetric systems // Methods Funct. Anal. Topology, 19 (2013), No. 3, 268-292.

[32] V. I. Mogilevskii, On spectral and pseudospectral functions of first- order symmetric systems // Ufa Math. Journal, 7 (2015), No. 2, 115-136. 
[33] V. I. Mogilevskii, Characteristic matrices and spectral functions of first order symmetric systems with maximal deficiency index of the minimal relation // Methods Funct. Anal. Topology, 21 (2015), No. 1, 76-98.

[34] V. I. Mogilevskii, Spectral and pseudospectral functions of Hamiltonian systems: development of the results by Arov-Dym and Sakhnovich // Methods Funct. Anal. Topology, 21 (2015), No. 4, 370-402.

[35] V. Mogilevskii, Spectral and pseudospectral functions of various dimensions for symmetric systems // J. Math. Sci., 221 (2017), No. 5, 679-711.

[36] A. L. Sakhnovich, Spectral functions of a canonical system of order $2 n$ // Mat. Sb. 181 (1990), No. 11, 1510-1524 (in Russian); transl. Math. USSR-Sb., 71 (1992), No. 2, 355-369.

[37] A. L. Sakhnovich, L. A. Sakhnovich, and I. Ya. Roitberg, Inverse problems and nonlinear evolution equations. Solutions, Darboux matrices and WeylTitchmarsh functions, De Gruyter Studies in Mathematics 47. De Gruyter, Berlin, 2013.

[38] A. V. S̆traus, On the extensions and the characteristic function of a symmetric operator // Izv. Akad. Nauk. SSSR, Ser.Mat., 32 (1968), No. 1, 186-207.

\section{CONTACT INFORMATION}

Vadim Mogilevskii Poltava V.G. Korolenko

National Pedagogical University,

Poltava, Ukraine

E-Mail: vadim.mogilevskii@gmail.com 